\title{
Combined bending and shear behaviour of slotted perforated steel channels: Numerical studies
}

\author{
N. Degtyareva \\ Institute of Architecture and Construction, South Ural State University, \\ Chelyabinsk, Russia. \\ P. Gatheeshgar \\ Faculty of Engineering and Environment, Northumbria University, \\ Newcastle, UK. \\ K. Poologanathan \\ Faculty of Engineering and Environment, Northumbria University, \\ Newcastle, UK. \\ S. Gunalan \\ School of Engineering and Built Environment, Griffith University \\ Gold Coast, QLD, 4222, Australia.
}

\section{Lawson}

Department of Civil and Environmental Engineering, University of Surrey, Surrey, UK.

\section{P. Sunday}

Department of Civil and Environmental Engineering, Imperial College London, London, UK.

\begin{abstract}
Cold-formed steel studs and purlins with staggered slotted perforations in webs are used in building structures to produce a better thermal performance of the profiles and for the energy efficiency of structures. On the other hand, the slotted webs result in an unfavourable effect in terms of the structural performance of the element, prominently their shear, bending and combined bending and shear strengths. Relatively little research has been reported on this subject despite its importance. Many research studies have been undertaken to examine the behaviour of conventional cold-formed steel (CFS) channel sections subject to combined bending and shear. To date, however, no research has been carried out to investigate how CFS channels with staggered slotted perforations behave under combined bending and shear actions. An extensive study on this area is therefore essential. Finite element (FE) models of CFS channels with staggered slotted perforations were developed to investigate their combined bending and shear capacity. A parametric study was conducted in detail by developing FE models based on the validation process with available experimental data. This paper presents the FE analysis details of CFS channels with staggered slotted perforations subject to combined
\end{abstract}


bending and shear actions and the FE results. New design equations were also proposed to predict the combined bending and shear capacity of steel channels with staggered slotted perforations.

Keywords: Cold-formed steel, Channel with Staggered Slotted Perforation, Combined Bending and Shear, Finite Element Analyses

\section{Introduction}

In recent times, the use of sophisticated manufacturing technologies and high strength steels has led to increased levels of cold-formed steel (CFS) construction using a range of thin-walled steel sections. CFS channels having slotted webs (see Figure 1) have been employed in residential and commercial buildings in order to enhance the response to changes in temperature of the profiles and to the enhance the energy efficiency of buildings [1-3]. Figure 2 shows the application of CFS channels with staggered slotted perforations. Slotted steel profiles have over the past years become a widely used section in building construction due to moisture resistance, lightweight, faster assembly, and good sound performance. The main difference for slotted steel profiles compared to non-slotted steel profiles is the reduced thermal conductivity, which is beneficial in terms of heat transfer (see Figure 3). On the other hand, the slotted steel profiles reduce the load-bearing capacity in bending and shear and add to deflections over solid sections.

Researches on CFS channel sections with the slotted web have been conducted in the past. The distortional and local buckling behaviour of compression members having staggered perforated webs were investigated and design proposals also presented to deal with web-perforated steel wall studs [4]. The shear strength and behaviour of CFS channels with slotted webs were investigated in [5-6] via numerical and experimental analyses and about $50-70 \%$ of the reduction in shear capacity was observed over the solid web channels. In addition, it was found that the shear strength of slotted web channels is hugely relying on the nature of the boundary conditions compared to the solid web channels [6]. Moreover, the behaviour of CFS channels with slotted webs under bending was investigated experimentally with a series of four-point loading structural test and results were checked with the direct strength method equations [7]. Combined bending and shear performance of a wide range of CFS sections including Csections, Z- sections, and hollow flange sections were studied experimentally in many research studies [8-13]. However, the research on the combined bending and shear behaviour of CFS channels with slotted webs remains unresearched. Therefore, a comprehensive numerical 
investigation was undertaken to examine the combined bending and shear behaviour of CFS channels having slotted web perforations in detail.

This paper provides information on the numerical studies of CFS channels with slotted web experiencing combined bending and shear action and the analysis of results. The simulation was executed with the general purpose finite element (FE) software, ANSYS [14]. Twelve solid web specimens were first modelled and the results were compared with available experimental results to validate the model. Then, the validated model was used to conduct a parametric study. A wide range of FE results obtained using ANSYS software was then used to develop a reduction factor formula to predict the combined bending and shear capacity of the channel sections with staggered perforated webs. The reduction factor formula is proposed as a function of section parameters and properties of the channel section.

\section{Numerical investigation}

The numerical investigation was performed using a general-purpose FE software, ANSYS. It has the ability to simulate the behaviour of cold-formed steel with material and geometrical non-linearities. The simulation process is conducted based on two steps, elastic buckling analysis and then followed by non-linear analysis. The wide range of information on the selection of element type, mesh refinement, boundary conditions, geometric imperfections, a method of analysis and validation is presented in the following subsections.

\subsection{Element type and material properties}

Shell element, named SHELL 181, was used to model the channels and web side plates (WSPs). This type of shell element is a four-node shell element with six degrees of freedom at each node and it has a capability of analysing thin-walled CFS elements allowing material and geometrical nonlinearities. The bi-linear isotropic hardening material model (BISO) was used to model the channels as the elastic-perfectly plastic behaviour model. CONTAT173 and TARGET170 elements were used for modelling contact between the web and web side plates. The web side plates were assumed as an elastic material. The modulus of elasticity and Poisson's ratio of the channels and WSPs were considered as $200 \mathrm{GPa}$ and 0.3 , respectively. Figure 4 depicts the constructed FE models of solid channels and slotted channels. 
2.2 Finite element mesh refinement

The channels and WSPs were refined with quadrilateral element meshes. Solid channels were modelled with the maximum mesh size of $5 \mathrm{~mm}$. The longitudinal direction in the perforated region, as well as the non-perforated regions of the slotted channels, were provided with a mesh refinement of $5 \mathrm{~mm}$. However, $1.5 \mathrm{~mm}$ of maximum element size in the vertical direction was provided for perforated regions as the meshing can directly govern the accuracy of the FE results as well as the rate of convergence. The same mesh density of the slotted channels was used in the study [6].

\subsection{Load and boundary conditions}

Figures 5 and 6 show the boundary conditions applied to channels without and with slotted webs channels which are experiencing combined bending and shear actions. Due to the symmetrical nature of the combined bending and shear three-point loading arrangement, half of the test set-up was modelled using symmetry boundary conditions. For modelling web side plates only from one side of the channel web surface-to-surface standard contact was defined between the web side plates and channel in the FE models for validation. The web side plates were the target surfaces and the channel was the contact surface. All WSP nodes are restrained in $\mathrm{x}$-direction. Web side plates from both sides of the channel web were used in the parametric study. WSP nodes and channel nodes adjacent to WSP are restrained in x-direction. The WSPs were connected with the channels by coupling the vertical and horizontal displacements of the nodes of the WSP and the channel at the bolt locations. Torsion/distortion restraint straps were modelled by restraining the translation in the direction perpendicular to the channel web and coupling the vertical displacements of the nodes of the top and bottom flanges at the locations where the straps were connected with channels in the tests. The translation of the support WSP in the vertical direction was restrained at the node located in the middle of the bottom edge of the plate. Vertical displacements of the nodes at the top edge of the WSP at the loading point were coupled. The models were loaded by an imposed displacement applied in small increments to one of the nodes at the WSP edge with the coupled vertical displacements.

\subsection{Initial geometric imperfections}

CFS has vulnerable behaviour of initial imperfections and this behaviour claims a high sensitivity to capacity predictions. To accommodate this, the initial geometric imperfections were added to the finite element models using eigenvalue buckling analysis. The lowest buckling modes are generally identified as critical modes. Therefore, the initial geometric 
imperfections were incorporated by modifying the geometry of the model from the lowest elastic buckling mode as obtained in previous linear elastic buckling analysis. An imperfection magnitude of $0.15 t$ was used to introduce imperfections for all models where $t$ is thickness. The justification of this selected magnitude is described in section 2.6.

\subsection{Method of analysis}

Linear elastic buckling analysis was conducted to obtain elastic buckling modes for models of slotted channels. The first elastic buckling mode was used to input the initial geometric imperfections for nonlinear analysis. The nonlinear static analysis was executed to obtain the combined bending and shear capacity and the failure shape of the model. The non-linear analysis considers the effects of large deformations and material yielding, which are vulnerable when CFS experiencing an application of load. The sparse direct equation solver was selected for the nonlinear analysis.

\subsection{FE Model validation}

In order to confirm the accuracy to predict the capacity of CFS channel first, twelve CFS solid web channels were validated with the available combined bending and shear experimental data presented in [11]. During this validation process, the capacities were obtained by incorporating four different options of imperfection magnitude values $(-0.64 t, 0.64 t,-0.15 t$ and $0.15 t$, where t refers to the thickness of the CFS channel). Table 1 describes the combined bending and shear capacities obtained from the experiments and from the FE analysis incorporating four different imperfection magnitude options. Pham and Hancock [11] conducted three-point loading combined bending and shear tests using back-to-back experimental set-ups. The ultimate shear load for a single channel is one-fourth of the applied load. Since only one channel was modelled for numerical investigation, the experimental values presented in Table 1 are the one-fourth of the values reported in [11]. These shear capacities are the ultimate shear capacity values on the load vs vertical displacement curves. From Table 1, it can be noticed that the mean value of the ratio (test to FE prediction results) of twelve specimens for $0.64 t,-0.64 t, 0.15 t$ and $-0.15 t$ options are 1.06, 1.00, 1.03 and 1.00, respectively. In addition, the coefficient of variation (COV) values are $0.075,0.066,0.072$ and 0.059 , respectively. These mean and COV values elaborate that the results of ANSYS [14] are not considerably sensitive to the initial imperfections. Similar conclusion was also reported in [15] for combined bending and shear numerical modelling. As a common approach, for further investigation (in parametric studies) the positive magnitude of $0.15 t$ was used as imperfection magnitude. 
161 Load-deflection plots obtained for the $200 \mathrm{~mm}$ deep channel with a thickness of $1.9 \mathrm{~mm}$ and 162 with straps (C20019-MV1) from experiments [11] and FE analysis are compared in Figure 7 163 while Figure 8 illustrates the failure mode of the C20019-MV1 with the solid web from 164 experiment and FE analysis. A high similarity between the failure shape in the experiment and 165 FE simulation was noticed. These comparisons confirm that FE analysis results show a 166 satisfactory agreement with corresponding experimental results of solid channels subject to 167 combined bending and shear action. It should be noted that in Figure 7 the vertical displacement 168 is higher to reach the ultimate load comparing to the FE prediction. This is because it is hard 169 to simulate the initial slip of the connecting bolts of the WSP through FE analysis which 170 commonly occurs in the experiments. Similar model characteristics were used to perform the 171 parametric studies of staggered slotted perforated sections subject to combined bending and 172 shear.

173 Table 1: Comparison of FE results and experimental [11] combined bending and shear capacities of solid 174 channels

\begin{tabular}{|c|c|c|c|c|c|c|c|c|c|}
\hline \multirow[t]{2}{*}{ Sections } & \multirow{2}{*}{$\begin{array}{l}\text { Test } \\
(\mathrm{kN})\end{array}$} & \multicolumn{8}{|c|}{ FE results for different imperfection magnitudes } \\
\hline & & $\begin{array}{l}\mathrm{FE}_{(0.64 \mathrm{t})} \\
(\mathrm{kN})\end{array}$ & $\begin{array}{l}\text { Test/ } \\
\mathrm{FE}_{(0.64 \mathrm{t})}\end{array}$ & $\begin{array}{l}\mathrm{FE}_{(-0.64 \mathrm{t})} \\
(\mathrm{kN})\end{array}$ & $\begin{array}{l}\text { Test/ } \\
\mathrm{FE}_{(-0.64 \mathrm{t})}\end{array}$ & $\begin{array}{l}\mathrm{FE}_{(0.15 \mathrm{t})} \\
(\mathrm{kN})\end{array}$ & $\begin{array}{l}\text { Test/ } \\
\mathrm{FE}_{(0.15 \mathrm{t})}\end{array}$ & $\begin{array}{l}\mathrm{FE}_{(-0.15 \mathrm{t})} \\
(\mathrm{kN})\end{array}$ & $\begin{array}{l}\text { Test/ } \\
\mathrm{FE}_{(-0.15 \mathrm{t})}\end{array}$ \\
\hline C15015-MV1 & 34.91 & 36.92 & 0.95 & 33.91 & 1.03 & 37.41 & 0.93 & 35.55 & 0.98 \\
\hline C15015-MVw & 26.80 & 24.36 & 1.10 & 27.83 & 0.96 & 25.02 & 1.07 & 28.44 & 0.94 \\
\hline C15019-MV1 & 48.37 & 48.85 & 0.99 & 50.53 & 0.96 & 50.89 & 0.95 & 50.94 & 0.95 \\
\hline C15019-MVw & 38.41 & 35.10 & 1.09 & 39.44 & 0.97 & 36.47 & 1.05 & 38.51 & 1.00 \\
\hline C15024-MV1 & 63.99 & 57.65 & 1.11 & 57.65 & 1.11 & 57.72 & 1.11 & 57.69 & 1.11 \\
\hline C15024-MVw & 54.18 & 45.23 & 1.20 & 48.31 & 1.12 & 46.91 & 1.15 & 47.84 & 1.13 \\
\hline C20015-MV1 & 34.22 & 36.28 & 0.94 & 34.18 & 1.00 & 36.82 & 0.93 & 35.00 & 0.98 \\
\hline C20015-MVw & 24.25 & 24.13 & 1.00 & 27.54 & 0.88 & 24.38 & 0.99 & 24.55 & 0.99 \\
\hline C20019-MV1 & 54.51 & 51.73 & 1.05 & 54.67 & 1.00 & 54.17 & 1.01 & 56.07 & 0.97 \\
\hline C20019-MVw & 39.80 & 35.32 & 1.13 & 41.31 & 0.96 & 36.22 & 1.10 & 40.92 & 0.97 \\
\hline C20024-MV1 & 72.47 & 70.72 & 1.02 & 71.77 & 1.01 & 72.80 & 1.00 & 72.85 & 0.99 \\
\hline C20024-MVw & 55.30 & 49.23 & 1.12 & 56.14 & 0.98 & 51.34 & 1.08 & 54.57 & 1.01 \\
\hline Min & & & 0.94 & & 0.88 & & 0.93 & & 0.94 \\
\hline Max & & & 1.20 & & 1.12 & & 1.15 & & 1.13 \\
\hline Mean & & & 1.06 & & 1.00 & & 1.03 & & 1.00 \\
\hline $\mathrm{COV}$ & & & 0.075 & & 0.066 & & 0.072 & & 0.059 \\
\hline
\end{tabular}

$175 \quad$ Note: $\mathrm{t}=$ thickness

\section{Parametric studies}

177 This section elaborates the details of the parametric study on the combined bending and shear

178 behaviour of channels with slotted webs. 


\subsection{Aims of the parametric study}

180 The aims of the parametric study are to investigate the combined bending and shear behaviour 181 of channels with slotted webs and develop either new or improved design guidelines or both 182 for them in order to predict their combined bending and shear capacities effectively and 183 increase their applicability in the construction sector.

\subsection{Varying parameters}

185

186

187

188

189

190

191

192

193

194

195

196

197

198

199

200

201

After the validation of the developed FE models as stated in the previous sections, a detailed parametric study was carried out based on the validated model to create a wide range of combined bending and shear capacity results database. Then, these results were adopted to develop modified design formulae for channels with slotted webs subjected to combined bending and shear action. In order to investigate the effect of section depth (d), thickness ( $t$ ), slot length $\left(\mathrm{L}_{\mathrm{sl}}\right)$, slot width $\left(\mathrm{W}_{\mathrm{sl}}\right)$, number of slot rows (n), number of slot row groups $(\mathrm{N})$, yield strength $\left(f_{y}\right)$ and aspect ratio $\left(a=a_{1} / d_{1}\right.$, where $a_{1}=$ shear span and $d_{1}=$ depth of the flat portion of the web) on the combined bending and shear capacity, different section depths ( $d=150$ and $200 \mathrm{~mm})$, thicknesses $(\mathrm{t}=1$ and $2 \mathrm{~mm})$, slot lengths (Lsl = 60 and $75 \mathrm{~mm})$, slot widths (Wsl = 3 and $5 \mathrm{~mm}$ ), number of rows ( $\mathrm{n}=6$ for $150 \mathrm{~mm}$ section and $\mathrm{n}=6$ and 8 for $200 \mathrm{~mm}$ section) yield strengths (fy $=300$ and $500 \mathrm{MPa}$ ) and aspect ratios $(\mathrm{a}=1.0,1.5,2.0,3.0,4.0)$ were considered in the parametric study. The varying parameters are presented in Table 2 . Total of $480 \mathrm{FE}$ models, allowing varying the expected influencing parameters, were considered in the parametric study. Figure 9 shows the dimensions of the FE model of channels for parametric study.

Table 2: Details of the parametric study

\begin{tabular}{lcccccccc}
\hline $\begin{array}{l}\mathrm{d} \\
(\mathrm{mm})\end{array}$ & $\mathrm{a}$ & $\begin{array}{c}\mathrm{t} \\
(\mathrm{mm})\end{array}$ & $\begin{array}{c}\mathrm{L}_{\mathrm{sl}} \\
(\mathrm{mm})\end{array}$ & $\begin{array}{c}\mathrm{W}_{\mathrm{sl}} \\
(\mathrm{mm})\end{array}$ & $\mathrm{n}$ & $\mathrm{N}$ & $\begin{array}{c}\mathrm{f}_{\mathrm{y}} \\
(\mathrm{MPa})\end{array}$ & $\begin{array}{c}\text { Number of } \\
\text { models }\end{array}$ \\
\hline 150 & 1.0 & 1,2 & 60,75 & 3,5 & 6 & 1,2 & 300,500 & 32 \\
& 1.5 & 1,2 & 60,75 & 3,5 & 6 & 1,2 & 300,500 & 32 \\
& 2.0 & 1,2 & 60,75 & 3,5 & 6 & 1,2 & 300,500 & 32 \\
& 3.0 & 1,2 & 60,75 & 3,5 & 6 & 1,2 & 300,500 & 32 \\
Sub-total & 4.0 & 1,2 & 60,75 & 3,5 & 6 & 1,2 & 300,500 & 32 \\
& & & & & & & & 160 \\
200 & 1.0 & 1,2 & 60,75 & 3,5 & 6,8 & 1,2 & 300,500 & 64 \\
& 1.5 & 1,2 & 60,75 & 3,5 & 6,8 & 1,2 & 300,500 & 64 \\
& 2.0 & 1,2 & 60,75 & 3,5 & 6,8 & 1,2 & 300,500 & 64 \\
& 3.0 & 1,2 & 60,75 & 3,5 & 6,8 & 1,2 & 300,500 & 64 \\
Sub-total & 4.0 & 1,2 & 60,75 & 3,5 & 6,8 & 1,2 & 300,500 & 64 \\
Total & & & & & & & & 320 \\
\hline Netan & & & & & & & & 480 \\
\hline
\end{tabular}




\subsection{Model nomenclature}

205 Each model was labelled to express the combined bending and shear series of models. For example, 200-1-75-3-2-8-300-1.5 represents the model indication of depth $200 \mathrm{~mm}$, thickness

$2071 \mathrm{~mm}$, slot length $75 \mathrm{~mm}$, slot width $3 \mathrm{~mm}$, number of slot row group 2, number of slot rows

208 8, yield stress $300 \mathrm{MPa}$ and aspect ratio 1.5, respectively.

\section{Results analysis}

210 The combined bending and shear behaviour of CFS channels having slotted webs were 211 analysed using 480 parametric models. The critical buckling mode of the solid and slotted 212 channels is depicted in Figure 10. Figure 11 illustrates the von Mises stress distribution of the 213 slotted CFS channels with 1 and 2 slot groups, and solid channel sections while Figure 12 214 shows the corresponding failure modes at the peak load stage. Tables 3-7 present the parametric 215 study results for the slotted channel sections and corresponding capacities for solid web channel 216 sections for aspect ratio $\mathrm{a}=1.0,1.5,2.0,3.0$ and 4.0. Here, $\mathrm{MV}_{\mathrm{u}}$ and $\mathrm{MV}_{\mathrm{nl}}$ are the combined 217 bending and shear capacity of the solid and slotted channels, respectively. It is noteworthy to 218 mention that combined bending and shear capacity is the reaction force obtained at the end supports of the three-piont loading model. The results from parametric studies were compared with the combined bending and shear capacities of the solid web CFS channels having equal dimensions. To elaborate, combined bending and shear capacity of slotted CFS model 200-175-3-2-8-300-3 was compared with its corresponding solid model 200-1-0-0-0-0-300-3. Then the ratio between slotted model and the solid model was determined for 480 parametric models. Overall the ratio between slotted and solid sections varies from 0.2 to 0.9 , thus overall $10-80 \%$ capacity reduction has been occurred due to the staggered perforated slots in webs. It was noticed that the maximum capacity reduction occurred when the web area reduction was at maximum in aspect ratio equal to 1 . The maximum web area reduction occurrs when the $\mathrm{L}_{\mathrm{s}}$, $\mathrm{W}_{\mathrm{sl}}, \mathrm{N}$ and $\mathrm{n}$ parameters are at their maximum values. Figures 13-16 show the load-vertical displacement behaviour of a particular model with aspect ratios 1, 1.5, 3 and 4, respectively.

Figure 17 shows the load-deflection plots of solid (200-1-0-0-0-0-300) and slotted (200-1-753-1-8-300) channels with different aspect ratios. The variations of the reduction factors are plotted against considered influencing parameters in Figure 18. Moreover, when the aspect ratio is only considered as an influencing factor in combined bending and shear capacity, the capacity reductions are decreasing with the aspect ratio. Figure 19 shows the plot of the reduction factor $\left(\mathrm{q}_{\mathrm{s}}=\mathrm{MV}_{\mathrm{nl}} / \mathrm{MV}_{\mathrm{u}}\right)$ versus aspect ratios (a). Each data point in Figure 19 is the 
236 average reduction factor value of 96 parametric results for considered aspect ratios. Figure 19

237 showed that the staggered slotted perforations in the channel webs significantly reduced

238 combined bending and shear capacities of the channels $(67 \%, 65 \%, 62 \%, 52 \%, 43 \%$ capacity

239 reductions for aspect ratios 1.0, 1.5, 2.0, 3.0 and 4.0, respectively).

Table 3: FE result for the parametric study (aspect ratio $=1.0$ )

\begin{tabular}{|c|c|c|c|c|c|c|c|c|c|}
\hline No & $\begin{array}{c}\text { Channels with Slotted } \\
\text { Webs }\end{array}$ & $\begin{array}{l}\mathrm{MV}_{\mathrm{nl}} \\
(\mathrm{kN})\end{array}$ & $\begin{array}{l}\mathrm{MV}_{\mathrm{u}} \\
(\mathrm{kN})\end{array}$ & $\begin{array}{l}\mathrm{MV}_{\mathrm{nl}} \\
\mathrm{MV}_{\mathrm{u}}\end{array}$ & No & $\begin{array}{l}\text { Channels with Slotted } \\
\text { Webs }\end{array}$ & $\begin{array}{l}\mathrm{MV}_{\mathrm{nl}} \\
(\mathrm{kN})\end{array}$ & $\begin{array}{l}\mathrm{MV}_{\mathrm{u}} \\
(\mathrm{kN})\end{array}$ & $\begin{array}{l}\mathrm{MV}_{\mathrm{n} /} \\
\mathrm{MV}_{\mathrm{u}}\end{array}$ \\
\hline 1 & $150-1-60-3-1-6-300-1$ & 6.55 & 17.06 & 0.38 & 49 & $200-2-60-3-1-6-300-1$ & 21.44 & 43.85 & 0.49 \\
\hline 2 & $150-1-60-5-1-6-300-1$ & 5.34 & 17.06 & 0.31 & 50 & $200-2-60-5-1-6-300-1$ & 17.71 & 43.85 & 0.40 \\
\hline 3 & $150-1-75-3-1-6-300-1$ & 5.60 & 17.06 & 0.33 & 51 & $200-2-75-3-1-6-300-1$ & 17.43 & 43.85 & 0.40 \\
\hline 4 & $150-1-75-5-1-6-300-1$ & 4.53 & 17.06 & 0.27 & 52 & $200-2-75-5-1-6-300-1$ & 14.39 & 43.85 & 0.33 \\
\hline 5 & $150-1-60-3-2-6-300-1$ & 6.12 & 17.06 & 0.36 & 53 & $200-2-60-3-2-6-300-1$ & 18.71 & 43.85 & 0.43 \\
\hline 6 & $150-1-60-5-2-6-300-1$ & 5.21 & 17.06 & 0.31 & 54 & $200-2-60-5-2-6-300-1$ & 15.82 & 43.85 & 0.36 \\
\hline 7 & $150-1-75-3-2-6-300-1$ & 4.83 & 17.06 & 0.28 & 55 & $200-2-75-3-2-6-300-1$ & 14.79 & 43.85 & 0.34 \\
\hline 8 & $150-1-75-5-2-6-300-1$ & 3.81 & 17.06 & 0.22 & 56 & $200-2-75-5-2-6-300-1$ & 12.20 & 43.85 & 0.28 \\
\hline 9 & $150-2-60-3-1-6-300-1$ & 17.89 & 39.09 & 0.46 & 57 & $200-2-60-3-1-8-300-1$ & 19.54 & 43.85 & 0.45 \\
\hline 10 & $150-2-60-5-1-6-300-1$ & 15.01 & 39.09 & 0.38 & 58 & $200-2-60-5-1-8-300-1$ & 14.96 & 43.85 & 0.34 \\
\hline 11 & $150-2-75-3-1-6-300-1$ & 13.87 & 39.09 & 0.35 & 59 & $200-2-75-3-1-8-300-1$ & 15.42 & 43.85 & 0.35 \\
\hline 12 & $150-2-75-5-1-6-300-1$ & 12.07 & 39.09 & 0.31 & 60 & $200-2-75-5-1-8-300-1$ & 12.77 & 43.85 & 0.29 \\
\hline 13 & $150-2-60-3-2-6-300-1$ & 16.78 & 39.09 & 0.43 & 61 & $200-2-60-3-2-8-300-1$ & 16.35 & 43.85 & 0.37 \\
\hline 14 & $150-2-60-5-2-6-300-1$ & 13.89 & 39.09 & 0.36 & 62 & $200-2-60-5-2-8-300-1$ & 13.24 & 43.85 & 0.30 \\
\hline 15 & $150-2-75-3-2-6-300-1$ & 11.69 & 39.09 & 0.30 & 63 & $200-2-75-3-2-8-300-1$ & 13.18 & 43.85 & 0.30 \\
\hline 16 & $150-2-75-5-2-6-300-1$ & 10.15 & 39.09 & 0.26 & 64 & $200-2-75-5-2-8-300-1$ & 10.95 & 43.85 & 0.25 \\
\hline 17 & $150-1-60-3-1-6-500-1$ & 9.91 & 24.69 & 0.40 & 65 & $200-1-60-3-1-6-500-1$ & 11.73 & 27.11 & 0.43 \\
\hline 18 & $150-1-60-5-1-6-500-1$ & 7.95 & 24.69 & 0.32 & 66 & $200-1-60-5-1-6-500-1$ & 9.77 & 27.11 & 0.36 \\
\hline 19 & $150-1-75-3-1-6-500-1$ & 8.77 & 24.69 & 0.36 & 67 & $200-1-75-3-1-6-500-1$ & 10.51 & 27.11 & 0.39 \\
\hline 20 & $150-1-75-5-1-6-500-1$ & 7.14 & 24.69 & 0.29 & 68 & $200-1-75-5-1-6-500-1$ & 8.99 & 27.11 & 0.33 \\
\hline 21 & $150-1-60-3-2-6-500-1$ & 8.93 & 24.69 & 0.36 & 69 & $200-1-60-3-2-6-500-1$ & 10.41 & 27.11 & 0.38 \\
\hline 22 & $150-1-60-5-2-6-500-1$ & 7.06 & 24.69 & 0.29 & 70 & $200-1-60-5-2-6-500-1$ & 8.58 & 27.11 & 0.32 \\
\hline 23 & $150-1-75-3-2-6-500-1$ & 7.49 & 24.69 & 0.30 & 71 & $200-1-75-3-2-6-500-1$ & 9.17 & 27.11 & 0.34 \\
\hline 24 & $150-1-75-5-2-6-500-1$ & 5.95 & 24.69 & 0.24 & 72 & $200-1-75-5-2-6-500-1$ & 7.31 & 27.11 & 0.27 \\
\hline 25 & $150-2-60-3-1-6-500-1$ & 28.78 & 63.11 & 0.46 & 73 & $200-1-60-3-1-8-500-1$ & 10.17 & 27.11 & 0.38 \\
\hline 26 & $150-2-60-5-1-6-500-1$ & 23.84 & 63.11 & 0.38 & 74 & $200-1-60-5-1-8-500-1$ & 8.47 & 27.11 & 0.31 \\
\hline 27 & $150-2-75-3-1-6-500-1$ & 21.87 & 63.11 & 0.35 & 75 & $200-1-75-3-1-8-500-1$ & 9.08 & 27.11 & 0.33 \\
\hline 28 & $150-2-75-5-1-6-500-1$ & 18.28 & 63.11 & 0.29 & 76 & $200-1-75-5-1-8-500-1$ & 7.88 & 27.11 & 0.29 \\
\hline 29 & $150-2-60-3-2-6-500-1$ & 26.37 & 63.11 & 0.42 & 77 & $200-1-60-3-2-8-500-1$ & 9.50 & 27.11 & 0.35 \\
\hline 30 & $150-2-60-5-2-6-500-1$ & 21.26 & 63.11 & 0.34 & 78 & $200-1-60-5-2-8-500-1$ & 7.56 & 27.11 & 0.28 \\
\hline 31 & $150-2-75-3-2-6-500-1$ & 19.21 & 63.11 & 0.30 & 79 & $200-1-75-3-2-8-500-1$ & 7.56 & 27.11 & 0.28 \\
\hline 32 & $150-2-75-5-2-6-500-1$ & 16.03 & 63.11 & 0.25 & 80 & $200-1-75-5-2-8-500-1$ & 5.82 & 27.11 & 0.21 \\
\hline 33 & $200-1-60-3-1-6-300-1$ & 7.74 & 19.00 & 0.41 & 81 & $200-2-60-3-1-6-500-1$ & 31.38 & 73.92 & 0.42 \\
\hline 34 & $200-1-60-5-1-6-300-1$ & 6.54 & 19.00 & 0.34 & 82 & $200-2-60-5-1-6-500-1$ & 25.97 & 73.92 & 0.35 \\
\hline 35 & $200-1-75-3-1-6-300-1$ & 7.02 & 19.00 & 0.37 & 83 & $200-2-75-3-1-6-500-1$ & 26.46 & 73.92 & 0.36 \\
\hline 36 & $200-1-75-5-1-6-300-1$ & 5.85 & 19.00 & 0.31 & 84 & $200-2-75-5-1-6-500-1$ & 21.93 & 73.92 & 0.30 \\
\hline 37 & $200-1-60-3-2-6-300-1$ & 7.04 & 19.00 & 0.37 & 85 & $200-2-60-3-2-6-500-1$ & 29.26 & 73.92 & 0.40 \\
\hline 38 & $200-1-60-5-2-6-300-1$ & 5.76 & 19.00 & 0.30 & 86 & $200-2-60-5-2-6-500-1$ & 23.32 & 73.92 & 0.32 \\
\hline 39 & $200-1-75-3-2-6-300-1$ & 5.88 & 19.00 & 0.31 & 87 & $200-2-75-3-2-6-500-1$ & 22.73 & 73.92 & 0.31 \\
\hline 40 & $200-1-75-5-2-6-300-1$ & 4.69 & 19.00 & 0.25 & 88 & $200-2-75-5-2-6-500-1$ & 18.84 & 73.92 & 0.25 \\
\hline 41 & $200-1-60-3-1-8-300-1$ & 6.81 & 19.00 & 0.36 & 89 & $200-2-60-3-1-8-500-1$ & 28.70 & 73.92 & 0.39 \\
\hline 42 & $200-1-60-5-1-8-300-1$ & 5.70 & 19.00 & 0.30 & 90 & $200-2-60-5-1-8-500-1$ & 22.40 & 73.92 & 0.30 \\
\hline 43 & $200-1-75-3-1-8-300-1$ & 6.09 & 19.00 & 0.32 & 91 & $200-2-75-3-1-8-500-1$ & 23.48 & 73.92 & 0.32 \\
\hline 44 & $200-1-75-5-1-8-300-1$ & 5.08 & 19.00 & 0.27 & 92 & $200-2-75-5-1-8-500-1$ & 19.33 & 73.92 & 0.26 \\
\hline 45 & $200-1-60-3-2-8-300-1$ & 6.33 & 19.00 & 0.33 & 93 & $200-2-60-3-2-8-500-1$ & 25.69 & 73.92 & 0.35 \\
\hline 46 & $200-1-60-5-2-8-300-1$ & 4.92 & 19.00 & 0.26 & 94 & $200-2-60-5-2-8-500-1$ & 20.64 & 73.92 & 0.28 \\
\hline 47 & $200-1-75-3-2-8-300-1$ & 4.83 & 19.00 & 0.25 & 95 & $200-2-75-3-2-8-500-1$ & 19.36 & 73.92 & 0.26 \\
\hline 48 & $200-1-75-5-2-8-300-1$ & 3.72 & 19.00 & 0.20 & 96 & $200-2-75-5-2-8-500-1$ & 15.75 & 73.92 & 0.21 \\
\hline
\end{tabular}


Table 4: FE result for the parametric study (aspect ratio $=1.5$ )

\begin{tabular}{|c|c|c|c|c|c|c|c|c|c|}
\hline No & $\begin{array}{l}\text { Channels with Slotted } \\
\text { Webs }\end{array}$ & $\begin{array}{l}\mathrm{MV}_{\mathrm{nl}} \\
(\mathrm{kN})\end{array}$ & $\begin{array}{l}\mathrm{MV}_{\mathrm{u}} \\
(\mathrm{kN})\end{array}$ & $\begin{array}{l}\mathrm{MV}_{\mathrm{n} 1 /} \\
\mathrm{MV}_{\mathrm{u}}\end{array}$ & No & $\begin{array}{l}\text { Channels with Slotted } \\
\text { Webs }\end{array}$ & $\begin{array}{l}\mathrm{MV}_{\mathrm{nl}} \\
(\mathrm{kN})\end{array}$ & $\begin{array}{l}\mathrm{MV}_{\mathrm{u}} \\
(\mathrm{kN})\end{array}$ & $\begin{array}{l}\mathrm{MV}_{\mathrm{n} / /} \\
\mathrm{MV}_{\mathrm{u}}\end{array}$ \\
\hline 1 & $150-1-60-3-1-6-300-1.5$ & 4.77 & 13.22 & 0.36 & 49 & $200-2-60-3-1-6-300-1.5$ & 18.23 & 36.79 & 0.50 \\
\hline 2 & $150-1-60-5-1-6-300-1.5$ & 3.92 & 13.22 & 0.30 & 50 & $200-2-60-5-1-6-300-1.5$ & 15.00 & 36.79 & 0.41 \\
\hline 3 & $150-1-75-3-1-6-300-1.5$ & 4.23 & 13.22 & 0.32 & 51 & $200-2-75-3-1-6-300-1.5$ & 14.01 & 36.79 & 0.38 \\
\hline 4 & $150-1-75-5-1-6-300-1.5$ & 3.46 & 13.22 & 0.26 & 52 & $200-2-75-5-1-6-300-1.5$ & 11.51 & 36.79 & 0.31 \\
\hline 5 & $150-1-60-3-2-6-300-1.5$ & 4.47 & 13.22 & 0.34 & 53 & $200-2-60-3-2-6-300-1.5$ & 16.93 & 36.79 & 0.46 \\
\hline 6 & $150-1-60-5-2-6-300-1.5$ & 3.54 & 13.22 & 0.27 & 54 & $200-2-60-5-2-6-300-1.5$ & 13.55 & 36.79 & 0.37 \\
\hline 7 & $150-1-75-3-2-6-300-1.5$ & 3.93 & 13.22 & 0.30 & 55 & $200-2-75-3-2-6-300-1.5$ & 12.59 & 36.79 & 0.34 \\
\hline 8 & $150-1-75-5-2-6-300-1.5$ & 3.03 & 13.22 & 0.23 & 56 & $200-2-75-5-2-6-300-1.5$ & 10.26 & 36.79 & 0.28 \\
\hline 9 & $150-2-60-3-1-6-300-1.5$ & 13.96 & 31.66 & 0.44 & 57 & $200-2-60-3-1-8-300-1.5$ & 16.91 & 36.79 & 0.46 \\
\hline 10 & $150-2-60-5-1-6-300-1.5$ & 11.77 & 31.66 & 0.37 & 58 & $200-2-60-5-1-8-300-1.5$ & 13.56 & 36.79 & 0.37 \\
\hline 11 & $150-2-75-3-1-6-300-1.5$ & 10.89 & 31.66 & 0.34 & 59 & $200-2-75-3-1-8-300-1.5$ & 13.10 & 36.79 & 0.36 \\
\hline 12 & $150-2-75-5-1-6-300-1.5$ & 9.39 & 31.66 & 0.30 & 60 & $200-2-75-5-1-8-300-1.5$ & 10.29 & 36.79 & 0.28 \\
\hline 13 & $150-2-60-3-2-6-300-1.5$ & 12.84 & 31.66 & 0.41 & 61 & $200-2-60-3-2-8-300-1.5$ & 15.04 & 36.79 & 0.41 \\
\hline 14 & $150-2-60-5-2-6-300-1.5$ & 10.77 & 31.66 & 0.34 & 62 & $200-2-60-5-2-8-300-1.5$ & 12.33 & 36.79 & 0.34 \\
\hline 15 & $150-2-75-3-2-6-300-1.5$ & 9.82 & 31.66 & 0.31 & 63 & $200-2-75-3-2-8-300-1.5$ & 11.12 & 36.79 & 0.30 \\
\hline 16 & $150-2-75-5-2-6-300-1.5$ & 7.99 & 31.66 & 0.25 & 64 & $200-2-75-5-2-8-300-1.5$ & 9.19 & 36.79 & 0.25 \\
\hline 17 & $150-1-60-3-1-6-500-1.5$ & 7.31 & 18.73 & 0.39 & 65 & $200-1-60-3-1-6-500-1.5$ & 9.8 & 19.80 & 0.50 \\
\hline 18 & $150-1-60-5-1-6-500-1.5$ & 5.89 & 18.73 & 0.31 & 66 & $200-1-60-5-1-6-500-1.5$ & 8.25 & 19.80 & 0.42 \\
\hline 19 & $150-1-75-3-1-6-500-1.5$ & 6.61 & 18.73 & 0.35 & 67 & $200-1-75-3-1-6-500-1.5$ & 8.22 & 19.80 & 0.42 \\
\hline 20 & $150-1-75-5-1-6-500-1.5$ & 5.44 & 18.73 & 0.29 & 68 & $-1-6-500-1.5$ & 6.81 & 19.80 & 0.34 \\
\hline 21 & $150-1-60-3-2-6-500-1.5$ & 6.48 & 18.73 & 0.35 & 69 & $500-1.5$ & 9.68 & 19.80 & 0.49 \\
\hline 22 & $150-1-60-5-2-6-500-1.5$ & 5.27 & 18.73 & 0.28 & 70 & $200-1-60-5-2-6-500-1.5$ & 7.88 & 19.80 & 0.40 \\
\hline 23 & $150-1-75-3-2-6-500-1.5$ & 5.76 & 18.73 & 0.31 & 71 & $200-1-75-3-2-6-500-1.5$ & 7.77 & 19.80 & 0.39 \\
\hline 24 & $150-1-75-5-2-6-500-1.5$ & 4.71 & 18.73 & 0.25 & 72 & $200-1-75-5-2-6-500-1.5$ & 6.03 & 19.80 & 0.30 \\
\hline 25 & $150-2-60-3-1-6-500-1.5$ & 20.87 & 51.13 & 0.41 & 73 & $200-1-60-3-1-8-500-1.5$ & 9.59 & 19.80 & 0.48 \\
\hline 26 & $150-2-60-5-1-6-500-1.5$ & 17.63 & 51.13 & 0.34 & 74 & $200-1-60-5-1-8-500-1.5$ & 7.56 & 19.80 & 0.38 \\
\hline 27 & $150-2-75-3-1-6-500-1.5$ & 16.20 & 51.13 & 0.32 & 75 & $-3-1-8-500-1.5$ & 7.65 & 19.80 & 0.39 \\
\hline 28 & $150-2-75-5-1-6-500-1.5$ & 13.49 & 51.13 & 0.26 & 76 & $200-1-75-5-1-8-500-1.5$ & 6.17 & 19.80 & 0.31 \\
\hline 29 & $150-2-60-3-2-6-500-1.5$ & 19.38 & 51.13 & 0.38 & 77 & $200-1-60-3-2-8-500-1.5$ & 8.01 & 19.80 & 0.40 \\
\hline 30 & $150-2-60-5-2-6-500-1.5$ & 16.61 & 51.13 & 0.32 & 78 & $200-1-60-5-2-8-500-1.5$ & 6.45 & 19.80 & 0.33 \\
\hline 31 & $150-2-75-3-2-6-500-1.5$ & 15.35 & 51.13 & 0.30 & 79 & $200-1$ & 6.37 & 19.80 & 0.32 \\
\hline 32 & $150-2-75-5-2-6-500-1.5$ & 12.66 & 51.13 & 0.25 & 80 & $200-1-75-5-2-8-500-1.5$ & 4.98 & 19.80 & 0.25 \\
\hline 33 & $200-1-60-3-1-6-300-1.5$ & 6.73 & 14.28 & 0.47 & 81 & $200-2-60-3-1-6-500-1.5$ & 25.87 & 58.96 & 0.44 \\
\hline 34 & $200-1-60-5-1-6-300-1.5$ & 5.61 & 14.28 & 0.39 & 82 & $200-2-60-5-1-6-500-1.5$ & 22.13 & 58.96 & 0.38 \\
\hline 35 & $200-1-75-3-1-6-300-1.5$ & 5.36 & 14.28 & 0.3 & 83 & $500-1.5$ & 21.24 & 58.96 & 0.36 \\
\hline 36 & $200-1-75-5-1-6-300-1.5$ & 4.51 & 14.28 & 0.32 & 84 & $200-2-75-5-1-6-500-1.5$ & 17.57 & 58.96 & 0.30 \\
\hline 37 & $200-1-60-3-2-6-300-1.5$ & 6.47 & 14.28 & 0.45 & 85 & $200-2-60-3-2-6-500-1.5$ & 26.19 & 58.96 & 0.44 \\
\hline 38 & $200-1-60-5-2-6-300-1.5$ & 5.28 & 14.28 & 0.37 & 86 & $200-2-60-5-2-6-500-1.5$ & 21.09 & 58.96 & 0.36 \\
\hline 39 & $200-1-75-3-2-6-300-1.5$ & 5.07 & 14.28 & 0.3 & 87 & $200-2-75-3-2-6-500-1.5$ & 19.78 & 58.96 & 0.34 \\
\hline 40 & $200-1-75-5-2-6-300-1.5$ & 3.87 & 14.28 & 0.27 & 88 & $200-2-75-5-2-6-500-1.5$ & 16.05 & 58.96 & 0.27 \\
\hline 41 & $200-1-60-3-1-8-300-1.5$ & 6.29 & 14.28 & 0.44 & 89 & $200-2-60-3-1-8-500-1.5$ & 23.71 & 58.96 & 0.40 \\
\hline 42 & & 5.13 & 14.28 & 0.36 & 90 & $200-2-60-5-1-8-500-1.5$ & 20.13 & 58.96 & 0.34 \\
\hline 43 & $200-1-75-3-1-8-300-1.5$ & 4.96 & 14.28 & 0.35 & 91 & $200-2-75-3-1-8-500-1.5$ & 19.60 & 58.96 & 0.33 \\
\hline 44 & $200-1-75-5-1-8-300-1.5$ & 3.95 & 14.28 & 0.28 & 92 & $200-2-75-5-1-8-500-1.5$ & 15.68 & 58.96 & 0.27 \\
\hline 45 & $200-1-60-3-2-8-300-1.5$ & 5.50 & 14.28 & 0.39 & 93 & $200-2-60-3-2-8-500-1.5$ & 22.05 & 58.96 & 0.37 \\
\hline 46 & $200-1-60-5-2-8-300-1.5$ & 4.40 & 14.28 & 0.31 & 94 & $200-2-60-5-2-8-500-1.5$ & 18.70 & 58.96 & 0.32 \\
\hline 47 & $200-1-75-3-2-8-300-1.5$ & 4.20 & 14.28 & 0.29 & 95 & $200-2-75-3-2-8-500-1.5$ & 17.26 & 58.96 & 0.29 \\
\hline 48 & $200-1-75-5-2-8-300-1.5$ & 3.19 & 14.28 & 0.22 & 96 & $200-2-75-5-2-8-500-1.5$ & 14.05 & 58.96 & 0.24 \\
\hline
\end{tabular}


Table 5: FE result for the parametric study (aspect ratio $=2.0$ )

\begin{tabular}{|c|c|c|c|c|c|c|c|c|c|}
\hline No & $\begin{array}{c}\text { Channels with Slotted } \\
\text { Webs }\end{array}$ & $\begin{array}{l}\mathrm{MV}_{\mathrm{nl}} \\
(\mathrm{kN})\end{array}$ & $\begin{array}{l}\mathrm{MV}_{\mathrm{u}} \\
(\mathrm{kN})\end{array}$ & $\begin{array}{l}\mathrm{MV}_{\mathrm{n} 1 /} \\
\mathrm{MV}_{\mathrm{u}}\end{array}$ & No & $\begin{array}{l}\text { Channels with Slotted } \\
\text { Webs }\end{array}$ & $\begin{array}{l}\mathrm{MV}_{\mathrm{nl}} \\
(\mathrm{kN})\end{array}$ & $\begin{array}{l}\mathrm{MV}_{\mathrm{u}} \\
(\mathrm{kN})\end{array}$ & $\begin{array}{l}\mathrm{MV}_{\mathrm{n} / /} \\
\mathrm{MV}_{\mathrm{u}}\end{array}$ \\
\hline 1 & $150-1-60-3-1-6-300-2$ & 4.57 & 10.74 & 0.43 & 49 & $200-2-60-3-1-6-300-2$ & 16.42 & 30.32 & 0.54 \\
\hline 2 & $150-1-60-5-1-6-300-2$ & 3.64 & 10.74 & 0.34 & 50 & $200-2-60-5-1-6-300-2$ & 13.40 & 30.32 & 0.44 \\
\hline 3 & $150-1-75-3-1-6-300-2$ & 3.60 & 10.74 & 0.33 & 51 & $200-2-75-3-1-6-300-2$ & 12.27 & 30.32 & 0.40 \\
\hline 4 & $150-1-75-5-1-6-300-2$ & 2.89 & 10.74 & 0.27 & 52 & $200-2-75-5-1-6-300-2$ & 9.73 & 30.32 & 0.32 \\
\hline 5 & $150-1-60-3-2-6-300-2$ & 4.71 & 10.74 & 0.44 & 53 & $200-2-60-3-2-6-300-2$ & 14.99 & 30.32 & 0.49 \\
\hline 6 & $150-1-60-5-2-6-300-2$ & 3.82 & 10.74 & 0.36 & 54 & $200-2-60-5-2-6-300-2$ & 12.42 & 30.32 & 0.41 \\
\hline 7 & $150-1-75-3-2-6-300-2$ & 3.60 & 10.74 & 0.34 & 55 & $200-2-75-3-2-6-300-2$ & 11.62 & 30.32 & 0.38 \\
\hline 8 & $150-1-75-5-2-6-300-2$ & 2.74 & 10.74 & 0.26 & 56 & $200-2-75-5-2-6-300-2$ & 9.68 & 30.32 & 0.32 \\
\hline 9 & $150-2-60-3-1-6-300-2$ & 12.89 & 26.32 & 0.49 & 57 & $200-2-60-3-1-8-300-2$ & 14.64 & 30.32 & 0.48 \\
\hline 10 & $150-2-60-5-1-6-300-2$ & 10.75 & 26.32 & 0.41 & 58 & $200-2-60-5-1-8-300-2$ & 12.30 & 30.32 & 0.41 \\
\hline 11 & $150-2-75-3-1-6-300-2$ & 9.64 & 26.32 & 0.37 & 59 & $200-2-75-3-1-8-300-2$ & 11.05 & 30.32 & 0.36 \\
\hline 12 & $150-2-75-5-1-6-300-2$ & 7.91 & 26.32 & 0.30 & 60 & $200-2-75-5-1-8-300-2$ & 8.93 & 30.32 & 0.29 \\
\hline 13 & $150-2-60-3-2-6-300-2$ & 12.31 & 26.32 & 0.47 & 61 & $200-2-60-3-2-8-300-2$ & 14.10 & 30.32 & 0.47 \\
\hline 14 & $150-2-60-5-2-6-300-2$ & 9.86 & 26.32 & 0.37 & 62 & $200-2-60-5-2-8-300-2$ & 11.37 & 30.32 & 0.38 \\
\hline 15 & $150-2-75-3-2-6-300-2$ & 9.41 & 26.32 & 0.36 & 63 & $200-2-75-3-2-8-300-2$ & 10.40 & 30.32 & 0.34 \\
\hline 16 & $150-2-75-5-2-6-300-2$ & 7.95 & 26.32 & 0.30 & 64 & $200-2-75-5-2-8-300-2$ & 7.30 & 30.32 & 0.24 \\
\hline 17 & $150-1-60-3-1-6-500-2$ & 6.77 & 15.54 & 0.44 & 65 & $200-1-60-3-1-6-500-2$ & 9.00 & 15.73 & 0.57 \\
\hline 18 & $150-1-60-5-1-6-500-2$ & 5.56 & 15.54 & 0.36 & 66 & $-5-1-6-500-2$ & 7.18 & 15.73 & 0.46 \\
\hline 19 & $150-1-75-3-1-6-500-2$ & 5.59 & 15.54 & 0.36 & 67 & $200-1-75-3-1-6-500-2$ & 7.72 & 15.73 & 0.49 \\
\hline 20 & $150-1-75-5-1-6-500-2$ & 4.45 & 15.54 & 0.29 & 68 & $200-1-75-5-1-6-500-2$ & 6.31 & 15.73 & 0.40 \\
\hline 21 & $150-1-60-3-2-6-500-2$ & 6.75 & 15.54 & 0.43 & 69 & $200-1-60-3-2-6-500-2$ & 7.85 & 15.73 & 0.50 \\
\hline 22 & $150-1-60-5-2-6-500-2$ & 5.46 & 15.54 & 0.35 & 70 & $200-1-60-5-2-6-500-2$ & 6.13 & 15.73 & 0.39 \\
\hline 23 & $150-1-75-3-2-6-500-2$ & 5.59 & 15.54 & 0.36 & 71 & $200-1-75-3-2-6-500-2$ & 6.93 & 15.73 & 0.44 \\
\hline 24 & $150-1-75-5-2-6-500-2$ & 4.30 & 15.54 & 0.28 & 72 & $200-1-75-5-2-6-500-2$ & 5.45 & 15.73 & 0.35 \\
\hline 25 & $150-2-60-3-1-6-500-2$ & 17.86 & 42.63 & 0.42 & 73 & $-1-8-500-2$ & 7.71 & 15.73 & 0.49 \\
\hline 26 & $150-2-60-5-1-6-500-2$ & 16.16 & 42.63 & 0.38 & 74 & $200-1-60-5-1-8-500-2$ & 6.13 & 15.73 & 0.39 \\
\hline 27 & $150-2-75-3-1-6-500-2$ & 14.65 & 42.63 & 0.34 & 75 & $200-1-75-3-1-8-500-2$ & 6.45 & 15.73 & 0.41 \\
\hline 28 & $150-2-75-5-1-6-500-2$ & 11.94 & 42.63 & 0.28 & 76 & $200-1-75-5-1-8-500-2$ & 5.25 & 15.73 & 0.33 \\
\hline 29 & $150-2-60-3-2-6-500-2$ & 19.07 & 42.63 & 0.45 & 77 & $200-1-60-3-2-8-500-2$ & 7.69 & 15.73 & 0.49 \\
\hline 30 & $150-2-60-5-2-6-500-2$ & 15.44 & 42.63 & 0.36 & 78 & $200-1-60-5-2-8-500-2$ & 5.76 & 15.73 & 0.37 \\
\hline 31 & $150-2-75-3-2-6-500-2$ & 14.67 & 42.63 & 0.34 & 79 & $200-1-75-3-2-8-500-2$ & 6.20 & 15.73 & 0.39 \\
\hline 32 & $150-2-75-5-2-6-500-2$ & 12.14 & 42.63 & 0.28 & 80 & $200-1-75-5-2-8-500-2$ & 4.67 & 15.73 & 0.30 \\
\hline 33 & $200-1-60-3-1-6-300-2$ & 5.96 & 11.33 & 0.53 & 81 & $200-2-60-3-1-6-500-2$ & 22.64 & 48.47 & 0.47 \\
\hline 34 & $200-1-60-5-1-6-300-2$ & 4.87 & 11.33 & 0.43 & 82 & $200-2-60-5-1-6-500-2$ & 19.77 & 48.47 & 0.41 \\
\hline 35 & $200-1-75-3-1-6-300-2$ & 5.06 & 11.33 & 0.45 & 83 & $200-2-75-3-1-6-500-2$ & 19.01 & 48.47 & 0.39 \\
\hline 36 & $200-1-75-5-1-6-300-2$ & 4.09 & 11.33 & 0.36 & 84 & $200-2-75-5-1-6-500-2$ & 14.58 & 48.47 & 0.30 \\
\hline 37 & $200-1-60-3-2-6-300-2$ & 5.44 & 11.33 & 0.48 & 85 & $200-2-60-3-2-6-500-2$ & 20.72 & 48.47 & 0.43 \\
\hline 38 & $200-1-60-5-2-6-300-2$ & 4.27 & 11.33 & 0.38 & 86 & $200-2-60-5-2-6-500-2$ & 17.39 & 48.47 & 0.36 \\
\hline 39 & $200-1-75-3-2-6-300-2$ & 4.51 & 11.33 & 0.4 & 87 & $200-2-75-3-2-6-500-2$ & 17.64 & 48.47 & 0.36 \\
\hline 40 & $200-1-75-5-2-6-300-2$ & 3.52 & 11.33 & 0.31 & 88 & $200-2-75-5-2-6-500-2$ & 14.55 & 48.47 & 0.30 \\
\hline 41 & $200-1-60-3-1-8-300-2$ & 5.27 & 11.33 & 0.47 & 89 & $200-2-60-3-1-8-500-2$ & 19.94 & 48.47 & 0.41 \\
\hline 42 & $200-1-60-5-1-8-300-2$ & 4.17 & 11.33 & 0.37 & 90 & $200-2-60-5-1-8-500-2$ & 16.72 & 48.47 & 0.34 \\
\hline 43 & $200-1-75-3-1-8-300-2$ & 4.46 & 11.33 & 0.39 & 91 & $200-2-75-3-1-8-500-2$ & 16.91 & 48.47 & 0.35 \\
\hline 44 & $200-1-75-5-1-8-300-2$ & 3.48 & 11.33 & 0.31 & 92 & $200-2-75-5-1-8-500-2$ & 13.72 & 48.47 & 0.28 \\
\hline 45 & $200-1-60-3-2-8-300-2$ & 5.26 & 11.33 & 0.46 & 93 & $200-2-60-3-2-8-500-2$ & 20.43 & 48.47 & 0.42 \\
\hline 46 & $200-1-60-5-2-8-300-2$ & 4.11 & 11.33 & 0.36 & 94 & $200-2-60-5-2-8-500-2$ & 16.83 & 48.47 & 0.35 \\
\hline 47 & $200-1-75-3-2-8-300-2$ & 4.02 & 11.33 & 0.36 & 95 & $200-2-75-3-2-8-500-2$ & 16.13 & 48.47 & 0.33 \\
\hline 48 & $200-1-75-5-2-8-300-2$ & 3.00 & 11.33 & 0.27 & 96 & $200-2-75-5-2-8-500-2$ & 12.55 & 48.47 & 0.26 \\
\hline
\end{tabular}


Table 6: FE result for the parametric study (aspect ratio $=3.0$ )

\begin{tabular}{|c|c|c|c|c|c|c|c|c|c|}
\hline No & $\begin{array}{c}\text { Channels with Slotted } \\
\text { Webs }\end{array}$ & $\begin{array}{l}\mathrm{MV}_{\mathrm{nl}} \\
(\mathrm{kN})\end{array}$ & $\begin{array}{l}\mathrm{MV}_{\mathrm{u}} \\
(\mathrm{kN})\end{array}$ & $\begin{array}{l}\mathrm{MV}_{\mathrm{nl}} \\
\mathrm{MV}_{\mathrm{u}}\end{array}$ & No & $\begin{array}{l}\text { Channels with Slotted } \\
\text { Webs }\end{array}$ & $\begin{array}{l}\mathrm{MV}_{\mathrm{nl}} \\
(\mathrm{kN})\end{array}$ & $\begin{array}{l}\mathrm{MV}_{\mathrm{u}} \\
(\mathrm{kN})\end{array}$ & $\begin{array}{l}\mathrm{MV}_{\mathrm{nl}} \\
\mathrm{MV}_{\mathrm{u}}\end{array}$ \\
\hline 1 & $150-1-60-3-1-6-300-3$ & 4.08 & 7.51 & 0.54 & 49 & $200-2-60-3-1-6-300-3$ & 14.35 & 22.22 & 0.65 \\
\hline 2 & $150-1-60-5-1-6-300-3$ & 3.20 & 7.51 & 0.43 & 50 & $200-2-60-5-1-6-300-3$ & 11.71 & 22.22 & 0.53 \\
\hline 3 & $150-1-75-3-1-6-300-3$ & 3.22 & 7.51 & 0.43 & 51 & $200-2-75-3-1-6-300-3$ & 10.58 & 22.22 & 0.48 \\
\hline 4 & $150-1-75-5-1-6-300-3$ & 2.57 & 7.51 & 0.34 & 52 & $200-2-75-5-1-6-300-3$ & 8.45 & 22.22 & 0.38 \\
\hline 5 & $150-1-60-3-2-6-300-3$ & 4.39 & 7.51 & 0.58 & 53 & $200-2-60-3-2-6-300-3$ & 13.42 & 22.22 & 0.60 \\
\hline 6 & $150-1-60-5-2-6-300-3$ & 3.36 & 7.51 & 0.45 & 54 & $200-2-60-5-2-6-300-3$ & 11.17 & 22.22 & 0.50 \\
\hline 7 & $150-1-75-3-2-6-300-3$ & 3.00 & 7.51 & 0.40 & 55 & $200-2-75-3-2-6-300-3$ & 10.44 & 22.22 & 0.47 \\
\hline 8 & $150-1-75-5-2-6-300-3$ & 2.27 & 7.51 & 0.30 & 56 & $200-2-75-5-2-6-300-3$ & 8.63 & 22.22 & 0.39 \\
\hline 9 & $150-2-60-3-1-6-300-3$ & 11.10 & 19.54 & 0.57 & 57 & $200-2-60-3-1-8-300-3$ & 12.86 & 22.22 & 0.58 \\
\hline 10 & $150-1-60-5-1-6-300-3$ & 9.04 & 19.54 & 0.46 & 58 & $200-2-60-5-1-8-300-3$ & 10.78 & 22.22 & 0.48 \\
\hline 11 & $150-2-75-3-1-6-300-3$ & 8.09 & 19.54 & 0.41 & 59 & $200-2-75-3-1-8-300-3$ & 9.26 & 22.22 & 0.42 \\
\hline 12 & $150-2-75-5-1-6-300-3$ & 6.61 & 19.54 & 0.34 & 60 & $200-2-75-5-1-8-300-3$ & 7.22 & 22.22 & 0.32 \\
\hline 13 & $150-2-60-3-2-6-300-3$ & 10.48 & 19.54 & 0.54 & 61 & $200-2-60-3-2-8-300-3$ & 12.74 & 22.22 & 0.57 \\
\hline 14 & $150-2-60-5-2-6-300-3$ & 8.42 & 19.54 & 0.43 & 62 & $200-2-60-5-2-8-300-3$ & 10.28 & 22.22 & 0.46 \\
\hline 15 & $150-2-75-3-2-6-300-3$ & 7.79 & 19.54 & 0.40 & 63 & $200-2-75-3-2-8-300-3$ & 9.28 & 22.22 & 0.42 \\
\hline 16 & $150-2-75-5-2-6-300-3$ & 6.31 & 19.54 & 0.32 & 64 & $200-2-75-5-2-8-300-3$ & 7.60 & 22.22 & 0.34 \\
\hline 17 & $150-1-60-3-1-6-500-3$ & 6.09 & 10.56 & 0.58 & 65 & $200-1-60-3-1-6-500-3$ & 7.94 & 10.50 & 0.76 \\
\hline 18 & $150-1-60-5-1-6-500-3$ & 4.71 & 10.56 & 0.45 & 66 & $200-1-60-5-1-6-500-3$ & 6.10 & 10.50 & 0.58 \\
\hline 19 & $150-1-75-3-1-6-500-3$ & 4.96 & 10.56 & 0.47 & 67 & $200-1-75-3-1-6-500-3$ & 6.67 & 10.50 & 0.64 \\
\hline 20 & $150-1-75-5-1-6-500-3$ & 4.01 & 10.56 & 0.38 & 68 & $200-1-75-5-1-6-500-3$ & 5.43 & 10.50 & 0.52 \\
\hline 21 & $150-1-60-3-2-6-500-3$ & 6.28 & 10.56 & 0.59 & 69 & $200-1-60-3-2-6-500-3$ & 7.14 & 10.50 & 0.68 \\
\hline 22 & $150-1-60-5-2-6-500-3$ & 4.97 & 10.56 & 0.47 & 70 & $200-1-60-5-2-6-500-3$ & 5.37 & 10.50 & 0.51 \\
\hline 23 & $150-1-75-3-2-6-500-3$ & 4.64 & 10.56 & 0.44 & 71 & $200-1-75-3-2-6-500-3$ & 6.37 & 10.50 & 0.61 \\
\hline 24 & $150-1-75-5-2-6-500-3$ & 3.47 & 10.56 & 0.33 & 72 & $200-1-75-5-2-6-500-3$ & 4.81 & 10.50 & 0.46 \\
\hline 25 & $150-2-60-3-1-6-500-3$ & 15.24 & 31.67 & 0.48 & 73 & $200-1-60-3-1-8-500-3$ & 6.83 & 10.50 & 0.65 \\
\hline 26 & $150-2-60-5-1-6-500-3$ & 13.49 & 31.67 & 0.43 & 74 & $200-1-60-5-1-8-500-3$ & 4.99 & 10.50 & 0.48 \\
\hline 27 & $150-2-75-3-1-6-500-3$ & 12.76 & 31.67 & 0.40 & 75 & $200-1-75-3-1-8-500-3$ & 5.78 & 10.50 & 0.55 \\
\hline 28 & $150-2-75-5-1-6-500-3$ & 10.25 & 31.67 & 0.32 & 76 & $200-1-75-5-1-8-500-3$ & 4.53 & 10.50 & 0.43 \\
\hline 29 & $150-2-60-3-2-6-500-3$ & 14.58 & 31.67 & 0.46 & 77 & $200-1-60-3-2-8-500-3$ & 6.79 & 10.50 & 0.65 \\
\hline 30 & $150-2-60-5-2-6-500-3$ & 11.90 & 31.67 & 0.38 & 78 & $200-1-60-5-2-8-500-3$ & 5.06 & 10.50 & 0.48 \\
\hline 31 & $150-2-75-3-2-6-500-3$ & 11.89 & 31.67 & 0.38 & 79 & $200-1-75-3-2-8-500-3$ & 5.84 & 10.50 & 0.56 \\
\hline 32 & $150-2-75-5-2-6-500-3$ & 9.73 & 31.67 & 0.31 & 80 & $200-1-75-5-2-8-500-3$ & 4.36 & 10.50 & 0.42 \\
\hline 33 & $200-1-60-3-1-6-300-3$ & 5.26 & 7.83 & 0.67 & 81 & $200-2-60-3-1-6-500-3$ & 20.26 & 33.64 & 0.60 \\
\hline 34 & $200-1-60-5-1-6-300-3$ & 4.16 & 7.83 & 0.53 & 82 & $200-2-60-5-1-6-500-3$ & 17.06 & 33.64 & 0.51 \\
\hline 35 & $200-1-75-3-1-6-300-3$ & 4.37 & 7.83 & 0.56 & 83 & $200-2-75-3-1-6-500-3$ & 16.51 & 33.64 & 0.49 \\
\hline 36 & $200-1-75-5-1-6-300-3$ & 3.50 & 7.83 & 0.45 & 84 & $200-2-75-5-1-6-500-3$ & 13.31 & 33.64 & 0.40 \\
\hline 37 & $200-1-60-3-2-6-300-3$ & 4.82 & 7.83 & 0.62 & 85 & $200-2-60-3-2-6-500-3$ & 18.82 & 33.64 & 0.56 \\
\hline 38 & $200-1-60-5-2-6-300-3$ & 3.75 & 7.83 & 0.48 & 86 & $200-2-60-5-2-6-500-3$ & 15.26 & 33.64 & 0.45 \\
\hline 39 & $200-1-75-3-2-6-300-3$ & 4.11 & 7.83 & 0.52 & 87 & $200-2-75-3-2-6-500-3$ & 15.80 & 33.64 & 0.47 \\
\hline 40 & $200-1-75-5-2-6-300-3$ & 3.17 & 7.83 & 0.40 & 88 & $200-2-75-5-2-6-500-3$ & 12.72 & 33.64 & 0.38 \\
\hline 41 & $200-1-60-3-1-8-300-3$ & 4.69 & 7.83 & 0.60 & 89 & $200-2-60-3-1-8-500-3$ & 17.99 & 33.64 & 0.53 \\
\hline 42 & $200-1-60-5-1-8-300-3$ & 3.49 & 7.83 & 0.45 & 90 & $200-2-60-5-1-8-500-3$ & 14.23 & 33.64 & 0.42 \\
\hline 43 & $200-1-75-3-1-8-300-3$ & 3.77 & 7.83 & 0.48 & 91 & $200-2-75-3-1-8-500-3$ & 14.19 & 33.64 & 0.42 \\
\hline 44 & $200-1-75-5-1-8-300-3$ & 2.93 & 7.83 & 0.37 & 92 & $200-2-75-5-1-8-500-3$ & 11.14 & 33.64 & 0.33 \\
\hline 45 & $200-1-60-3-2-8-300-3$ & 4.54 & 7.83 & 0.58 & 93 & $200-2-60-3-2-8-500-3$ & 18.09 & 33.64 & 0.54 \\
\hline 46 & $200-1-60-5-2-8-300-3$ & 3.42 & 7.83 & 0.44 & 94 & $200-2-60-5-2-8-500-3$ & 13.90 & 33.64 & 0.41 \\
\hline 47 & $200-1-75-3-2-8-300-3$ & 3.74 & 7.83 & 0.48 & 95 & $200-2-75-3-2-8-500-3$ & 14.46 & 33.64 & 0.43 \\
\hline 48 & $200-1-75-5-2-8-300-3$ & 2.78 & 7.83 & 0.36 & 96 & $200-2-75-5-2-8-500-3$ & 11.37 & 33.64 & 0.34 \\
\hline
\end{tabular}

Note: $\mathrm{MV}_{\mathrm{nl}}=$ combined bending and shear capacity of a slotted web channel, $\mathrm{MV}_{\mathrm{u}}=$ combined bending and shear capacity of a solid web channel 
Table 7: FE result for the parametric study (aspect ratio $=4.0$ )

\begin{tabular}{|c|c|c|c|c|c|c|c|c|c|}
\hline No & $\begin{array}{c}\text { Channels with Slotted } \\
\text { Webs }\end{array}$ & $\begin{array}{l}\mathrm{MV}_{\mathrm{nl}} \\
(\mathrm{kN})\end{array}$ & $\begin{array}{l}\mathrm{MV}_{\mathrm{u}} \\
(\mathrm{kN})\end{array}$ & $\begin{array}{l}\mathrm{MV}_{\mathrm{n} /} \\
\mathrm{MV}_{\mathrm{u}}\end{array}$ & No & $\begin{array}{l}\text { Channels with Slotted } \\
\text { Webs }\end{array}$ & $\begin{array}{l}\mathrm{MV}_{\mathrm{nl}} \\
(\mathrm{kN})\end{array}$ & $\begin{array}{l}\mathrm{MV}_{\mathrm{u}} \\
(\mathrm{kN})\end{array}$ & $\begin{array}{l}\mathrm{MV}_{\mathrm{n} / /} \\
\mathrm{MV}_{\mathrm{u}}\end{array}$ \\
\hline 1 & $150-1-60-3-1-6-300-4$ & 3.76 & 5.77 & 0.65 & 49 & $200-2-60-3-1-6-300-4$ & 12.88 & 17.25 & 0.75 \\
\hline 2 & $150-1-60-5-1-6-300-4$ & 2.77 & 5.77 & 0.48 & 50 & $200-2-60-5-1-6-300-4$ & 10.60 & 17.25 & 0.61 \\
\hline 3 & $150-1-75-3-1-6-300-4$ & 3.17 & 5.77 & 0.55 & 51 & $200-2-75-3-1-6-300-4$ & 9.69 & 17.25 & 0.56 \\
\hline 4 & $150-1-75-5-1-6-300-4$ & 2.49 & 5.77 & 0.43 & 52 & $200-2-75-5-1-6-300-4$ & 7.72 & 17.25 & 0.45 \\
\hline 5 & $150-1-60-3-2-6-300-4$ & 3.65 & 5.77 & 0.63 & 53 & $200-2-60-3-2-6-300-4$ & 12.40 & 17.25 & 0.72 \\
\hline 6 & $150-1-60-5-2-6-300-4$ & 2.64 & 5.77 & 0.46 & 54 & $200-2-60-5-2-6-300-4$ & 10.09 & 17.25 & 0.58 \\
\hline 7 & $150-1-75-3-2-6-300-4$ & 3.02 & 5.77 & 0.52 & 55 & $200-2-75-3-2-6-300-4$ & 9.46 & 17.25 & 0.55 \\
\hline 8 & $150-1-75-5-2-6-300-4$ & 2.27 & 5.77 & 0.39 & 56 & $200-2-75-5-2-6-300-4$ & 7.39 & 17.25 & 0.43 \\
\hline 9 & $150-2-60-3-1-6-300-4$ & 10.63 & 15.51 & 0.69 & 57 & $200-2-60-3-1-8-300-4$ & 11.84 & 17.25 & 0.69 \\
\hline 10 & $150-2-60-5-1-6-300-4$ & 8.53 & 15.51 & 0.55 & 58 & $200-2-60-5-1-8-300-4$ & 9.62 & 17.25 & 0.56 \\
\hline 11 & $150-2-75-3-1-6-300-4$ & 7.71 & 15.51 & 0.50 & 59 & $200-2-75-3-1-8-300-4$ & 8.68 & 17.25 & 0.50 \\
\hline 12 & $150-2-75-5-1-6-300-4$ & 6.28 & 15.51 & 0.40 & 60 & $200-2-75-5-1-8-300-4$ & 6.62 & 17.25 & 0.38 \\
\hline 13 & $150-2-60-3-2-6-300-4$ & 10.39 & 15.51 & 0.67 & 61 & $200-2-60-3-2-8-300-4$ & 11.97 & 17.25 & 0.69 \\
\hline 14 & $150-2-60-5-2-6-300-4$ & 8.34 & 15.51 & 0.54 & 62 & $200-2-60-5-2-8-300-4$ & 9.54 & 17.25 & 0.55 \\
\hline 15 & $150-2-75-3-2-6-300-4$ & 7.58 & 15.51 & 0.49 & 63 & $200-2-75-3-2-8-300-4$ & 8.64 & 17.25 & 0.50 \\
\hline 16 & $150-2-75-5-2-6-300-4$ & 6.13 & 15.51 & 0.40 & 64 & $200-2-75-5-2-8-300-4$ & 6.56 & 17.25 & 0.38 \\
\hline 17 & $150-1-60-3-1-6-500-4$ & 5.64 & 8.06 & 0.70 & 65 & $200-1-60-3-1-6-500-4$ & 7.36 & 8.20 & 0.90 \\
\hline 18 & $150-1-60-5-1-6-500-4$ & 4.19 & 8.06 & 0.52 & 66 & $200-1-60-5-1-6-500-4$ & 5.78 & 8.20 & 0.70 \\
\hline 19 & $150-1-75-3-1-6-500-4$ & 4.92 & 8.06 & 0.61 & 67 & $200-1-75-3-1-6-500-4$ & 6.02 & 8.20 & 0.73 \\
\hline 20 & $150-1-75-5-1-6-500-4$ & 3.90 & 8.06 & 0.48 & 68 & $200-1-75-5-1-6-500-4$ & 4.89 & 8.20 & 0.60 \\
\hline 21 & $150-1-60-3-2-6-500-4$ & 5.23 & 8.06 & 0.65 & 69 & $200-1-60-3-2-6-500-4$ & 6.70 & 8.20 & 0.82 \\
\hline 22 & $150-1-60-5-2-6-500-4$ & 3.81 & 8.06 & 0.47 & 70 & $200-1-60-5-2-6-500-4$ & 4.88 & 8.20 & 0.59 \\
\hline 23 & $150-1-75-3-2-6-500-4$ & 4.65 & 8.06 & 0.58 & 71 & $200-1-75-3-2-6-500-4$ & 5.68 & 8.20 & 0.69 \\
\hline 24 & $150-1-75-5-2-6-500-4$ & 3.90 & 8.06 & 0.48 & 72 & $200-1-75-5-2-6-500-4$ & 4.40 & 8.20 & 0.54 \\
\hline 25 & $150-2-60-3-1-6-500-4$ & 15.32 & 25.10 & 0.61 & 73 & $200-1-60-3-1-8-500-4$ & 6.43 & 8.20 & 0.78 \\
\hline 26 & $150-2-60-5-1-6-500-4$ & 12.78 & 25.10 & 0.51 & 74 & $200-1-60-5-1-8-500-4$ & 4.55 & 8.20 & 0.55 \\
\hline 27 & $150-2-75-3-1-6-500-4$ & 12.01 & 25.10 & 0.48 & 75 & $200-1-75-3-1-8-500-4$ & 5.20 & 8.20 & 0.63 \\
\hline 28 & $150-2-75-5-1-6-500-4$ & 9.59 & 25.10 & 0.38 & 76 & $200-1-75-5-1-8-500-4$ & 4.05 & 8.20 & 0.49 \\
\hline 29 & $150-2-60-3-2-6-500-4$ & 15.17 & 25.10 & 0.60 & 77 & $200-1-60-3-2-8-500-4$ & 6.45 & 8.20 & 0.79 \\
\hline 30 & $150-2-60-5-2-6-500-4$ & 11.93 & 25.10 & 0.48 & 78 & $200-1-60-5-2-8-500-4$ & 4.62 & 8.20 & 0.56 \\
\hline 31 & $150-2-75-3-2-6-500-4$ & 11.76 & 25.10 & 0.47 & 79 & $200-1-75-3-2-8-500-4$ & 5.06 & 8.20 & 0.62 \\
\hline 32 & $150-2-75-5-2-6-500-4$ & 9.23 & 25.10 & 0.37 & 80 & $200-1-75-5-2-8-500-4$ & 4.08 & 8.20 & 0.50 \\
\hline 33 & $200-1-60-3-1-6-300-4$ & 4.83 & 5.90 & 0.82 & 81 & $200-2-60-3-1-6-500-4$ & 19.06 & 25.88 & 0.74 \\
\hline 34 & $200-1-60-5-1-6-300-4$ & 3.94 & 5.90 & 0.67 & 82 & $200-2-60-5-1-6-500-4$ & 15.29 & 25.88 & 0.59 \\
\hline 35 & $200-1-75-3-1-6-300-4$ & 3.97 & 5.90 & 0.67 & 83 & $200-2-75-3-1-6-500-4$ & 15.03 & 25.88 & 0.58 \\
\hline 36 & $200-1-75-5-1-6-300-4$ & 3.13 & 5.90 & 0.53 & 84 & $200-2-75-5-1-6-500-4$ & 12.08 & 25.88 & 0.47 \\
\hline 37 & $200-1-60-3-2-6-300-4$ & 4.48 & 5.90 & 0.76 & 85 & $200-2-60-3-2-6-500-4$ & 18.14 & 25.88 & 0.70 \\
\hline 38 & $200-1-60-5-2-6-300-4$ & 3.33 & 5.90 & 0.56 & 86 & $200-2-60-5-2-6-500-4$ & 13.92 & 25.88 & 0.54 \\
\hline 39 & $200-1-75-3-2-6-300-4$ & 3.86 & 5.90 & 0.65 & 87 & $200-2-75-3-2-6-500-4$ & 14.27 & 25.88 & 0.55 \\
\hline 40 & $200-1-75-5-2-6-300-4$ & 2.98 & 5.90 & 0.50 & 88 & $200-2-75-5-2-6-500-4$ & 11.55 & 25.88 & 0.45 \\
\hline 41 & $200-1-60-3-1-8-300-4$ & 4.24 & 5.90 & 0.72 & 89 & $200-2-60-3-1-8-500-4$ & 17.13 & 25.88 & 0.66 \\
\hline 42 & $200-1-60-5-1-8-300-4$ & 3.15 & 5.90 & 0.53 & 90 & $200-2-60-5-1-8-500-4$ & 13.01 & 25.88 & 0.50 \\
\hline 43 & $200-1-75-3-1-8-300-4$ & 3.47 & 5.90 & 0.59 & 91 & $200-2-75-3-1-8-500-4$ & 12.95 & 25.88 & 0.50 \\
\hline 44 & $200-1-75-5-1-8-300-4$ & 2.69 & 5.90 & 0.46 & 92 & $200-2-75-5-1-8-500-4$ & 10.20 & 25.88 & 0.39 \\
\hline 45 & $200-1-60-3-2-8-300-4$ & 4.32 & 5.90 & 0.73 & 93 & $200-2-60-3-2-8-500-4$ & 17.46 & 25.88 & 0.67 \\
\hline 46 & $200-1-60-5-2-8-300-4$ & 3.11 & 5.90 & 0.53 & 94 & $200-2-60-5-2-8-500-4$ & 13.29 & 25.88 & 0.51 \\
\hline 47 & $200-1-75-3-2-8-300-4$ & 3.49 & 5.90 & 0.59 & 95 & $200-2-75-3-2-8-500-4$ & 13.15 & 25.88 & 0.51 \\
\hline 48 & $200-1-75-5-2-8-300-4$ & 2.65 & 5.90 & 0.45 & 96 & $200-2-75-5-2-8-500-4$ & 10.36 & 25.88 & 0.40 \\
\hline
\end{tabular}




\section{Proposed design guidelines}

266 When a failure occurs due to the presence of both shear force and bending moment, thus

267 produce a notable effect on the strength of the section. This phenomenon is commonly studied

268 and expressed in an interaction diagram. Clause 3.3.5 of AS/NZ 4600 [16] and section C 3.3.2

269 of AISI [17] define the combined bending and shear actions of CFS beams with unstiffened

270 webs as:

271

$$
\left[\frac{M^{*}}{M_{S}}\right]^{2}+\left[\frac{V^{*}}{V_{V}}\right]^{2}=1
$$

272 where

$273 M^{*}=$ Applied bending moment,

$274 M_{s}=$ The section moment capacity under pure bending,

$275 \quad V^{*}=$ The applied shear force, and

$276 \quad V_{v}=$ The shear capacity under pure shear.

277 Moreover, the upper limit to account combined bending and shear actions for beams with

278 transverse web stiffeners are also provided in Clause 3.3.5 of AS/NZ 4600 [16] and section C

$279 \quad 3.3 .2$ of AISI [17]:

280

$$
0.6\left[\frac{M^{*}}{M_{s}}\right]+\left[\frac{V^{*}}{V_{v}}\right]=1.3
$$

281 It should be noted that here $\left(\mathrm{M}^{*} / \mathrm{M}_{\mathrm{s}}\right)>0.5$ and $\left(\mathrm{V}^{*} / \mathrm{V}_{\mathrm{v}}\right)>0.7$ to ensure both ratios do not exceed

282 1.0. These two equations (Equation 1 and Equation 2) are graphically illustrated in Figure 20.

283 Figure 20 was also plotted with the test results obtained from the research conducted by Pham

284 [18] investigating combined bending and shear behaviour of lipped channel beams without web

285 openings as well as SupaCee sections. However, the plotted points are test results of lipped

286 channel sections subject to bending, shear, and combined bending and shear, as this study

287 limited to CFS, lipped channel beams having slotted web. It can be noticed that the combined

288 bending and shear test results are closer to the interaction curve when the pure bending and

289 pure shear capacities are calculated using the Direct Strength Method (DSM). Here section

290 moment capacity in pure bending is replaced with local buckling moment capacity $\left(\mathrm{M}_{\mathrm{sl}}\right)$ as the

291 test specimens are allowed to fail due to local buckling stress with the provided straps in the

292 pure bending zone. The nominal shear capacity $\left(\mathrm{V}_{\mathrm{v}}\right)$ is obtained using DSM. The account for

293 the tension field action and using shear buckling coefficient for whole section instead of

294 considering web only in DSM approach to calculate $V_{v}$ made the test results reasonably close 
to the interaction curve. Further, the use of effective width method and AS/ NZ 4600 [16] for the calculation of Ms and Vv, respectively showed conservative results [18].

However, this study focused on proposing a reduction factor $\mathrm{q}_{\mathrm{s}}\left(=\mathrm{MV}_{\mathrm{n}} / \mathrm{MV}_{\mathrm{u}}\right)$ as a function of influencing parameters which contribute to the combined bending and shear capacity of slotted web CFS channels. Therefore, FE combined bending and shear capacities of the perforated and solid channels were directly compared.

The comparison showed that the staggered slotted perforations in the channel webs reduced combined bending and shear capacities of the channels. Current shear design rules for coldformed steel beams with web openings are based on a reduction factor $\left(\mathrm{q}_{\mathrm{s}}\right)$ defined as the ratio of the nominal shear capacity of CFS beams with web openings $\left(\mathrm{V}_{\mathrm{nl}}\right)$ to the nominal shear capacity of cold-formed steel beams without web openings $\left(\mathrm{V}_{\mathrm{u}}\right)$. Therefore, suitable design guidelines are also necessary to calculate combined bending and shear capacities of channels with slotted webs $\left(\mathrm{MV}_{\mathrm{nl}}\right)$ based on reduction factor $\left(\mathrm{q}_{\mathrm{s}}\right)$ and combined bending and shear capacity of cold-formed steel beams without web openings $\left(\mathrm{MV}_{\mathrm{u}}\right)$.

As a first approach, New equation (Equation 3) was proposed for reduction factor based on FEA results by only taking the aspect ratio into account as the governing factor of the capacity of perforated slotted CFS channels when experiencing combined bending and shear actions (see Figure 19). The mean value for the ratio between $\mathrm{q}_{\mathrm{s}}\left(=\mathrm{MV}_{\mathrm{nl}} / \mathrm{MV}_{\mathrm{u}}\right)$ and $\mathrm{q}_{\mathrm{s} 1}$ gives a mean value of 1.00 and COV of 0.19 for all 480 models.

$$
q_{s 1}=\frac{M V_{n l}}{M V_{u}}=0.01\left[\frac{a_{1}}{d_{1}}\right]^{2}+0.032\left[\frac{a_{1}}{d_{1}}\right]+0.28
$$

where

$q_{s l}=$ reduction factor for combined bending and shear capacity

$a_{1}=$ the shear span

$d_{1}=$ the depth of the channel

Further, an overall reduction factor $\mathrm{q}_{\mathrm{s} 2}$ is also proposed through this study which accounts for all the parameters considered in the parametric study. This reduction factor is formed as a function of d, t, Lsl, Wsl, n, N, fy and $\left(a_{1} / d_{1}\right)$. Equation 4 represents the overall reduction factor for staggered perforated slotted CFS channels subject to combined bending and shear actions. All the parameters were normalised with standard and relevant values (standard yield strength is taken as $250 \mathrm{MPa}$ ) and also with other limiting dimensions (see Figure 1). This overall reduction factor was developed using a genetic algorithm (Generalised Reduced Gradient 
(GRG) non-linear) solver. It can be noticed that the coefficients of the $\left(a_{1} / d_{1}\right)$ terms are different for Equation 3 and Equation 4. This variation is due to the influence of the other parameters in Equation 4 while those are not as part of Equation 3.

$$
\mathrm{q}_{\mathrm{s} 2}=\frac{\left[0.003\left[\frac{\mathrm{a}}{\mathrm{d}_{1}}\right]^{2}+0.004\left[\frac{\mathrm{a}_{1}}{\mathrm{~d}_{1}}\right]+0.078\right]\left[\frac{\mathrm{d}_{1}}{\mathrm{t}}\right]^{0.092}\left[\frac{\mathrm{f}}{250}\right]^{0.013}}{\left[\frac{\mathrm{L}_{\mathrm{sl}}}{100}\right]^{0.99}\left[\frac{\mathrm{W}_{\mathrm{sl}}}{9.5}\right]^{0.43}\left[\frac{\mathrm{N}}{\mathrm{n}}\right]^{0.068}}
$$

This reduction factor $\mathrm{q}_{\mathrm{s} 2}$ is capable to predict the combined bending and shear capacity of a staggered perforated slotted channel as it has been developed by maintaining the mean value equal to 1. To elaborate, the ratio between $\mathrm{q}_{\mathrm{s}}\left(=\mathrm{MV}_{\mathrm{n}} / \mathrm{MV}_{\mathrm{u}}\right)$ and $\mathrm{q}_{\mathrm{s} 2}$ gives a mean value of 1.00 and COV of 0.10 for 480 parametric models considered in this study. Figure 21 demonstrates the aforementioned ratio of 480 models, which are very much closer to unity. Therefore, considering COV values of Equation 3 ( $\mathrm{COV}$ is 0.19 ) and Equation 4 (COV is 0.10 ), it can be concluded that $\mathrm{q}_{\mathrm{s} 2}$ is a better solution which could help the designers to accurately predict the capacity of the staggered perforated slotted channels subject to combined bending and shear action. The FE results of the 480 models are plotted in Figure 22. In addition, combined bending and shear test results which are plotted in Figure 20 also plotted in the same figure. Figure 22 shows that the proposed design equations are able to predict the combined bending and shear capacity of slotted perforated channels.

\section{Concluding remarks}

This paper presents the details of the numerical studies of cold-formed steel channels with slotted webs subject to combined bending and shear action and the results of these analyses. A detailed parametric study was performed based on validated FE models in order to investigate the influencing parameters (section depth $(\mathrm{d})$, thickness $(\mathrm{t})$, slot length $\left(\mathrm{L}_{\mathrm{sl}}\right)$, slot width $\left(\mathrm{W}_{\mathrm{sl}}\right)$, number of slot rows (n), number of slot row groups $(N)$, yield strength $\left(f_{y}\right)$ and aspect ratio (a)) on the combined bending and shear behaviour of staggered slotted perforated steel channels. Subsequently, combined bending and shear capacities of the staggered slotted perforated steel channels were directly compared with the solid web steel channels with the same dimensions. The comparison showed that the staggered slotted perforations in the channel webs significantly reduced the combined bending and shear capacities. New design equations were proposed as a function of the influencing parameters based on the wide range of parametric study results. Therefore, combined bending and shear capacity of the staggered slotted 
357 perforated steel channels can be calculated with reasonable accuracy through the newly 358 proposed equations.

\section{Acknowledgements}

The authors of this paper would like to acknowledge South Ural State University and Northumbria University's support with necessary research facilities and technical support

\section{References}

[1] T. Höglund, H. Burstrand, Slotted steel studs to reduce thermal bridges in insulated walls, Thin-Walled Structures. 32 (1998) 81-109. doi:10.1016/s0263-8231(98)000287.

[2] R. LaBoube, Development of cost-effective, energy-efficient steel framing: Thermal performance of slit-web steel wall studs, American Iron and Steel Institute, 2006.

[3] J. Lipták-Váradi, Equivalent thermal conductivity of steel girders with slotted web, Periodica Polytechnica Civil Engineering. 54 (2010) 163. doi:10.3311/pp.ci.2010-2.12.

[4] Kesti, Local and distortional buckling of perforated steel wall studs, PhD, Helsinki University of Technology, 2000.

[5] N. Degtyareva, V. Degtyarev, Experimental investigation of cold-formed steel channels with slotted webs in shear, Thin-Walled Structures. 102 (2016) 30-42. doi:10.1016/j.tws.2016.01.012.

[6] V. Degtyarev, N. Degtyareva, Finite element modeling of cold-formed steel channels with solid and slotted webs in shear, Thin-Walled Structures. 103 (2016) 183-198. doi:10.1016/j.tws.2016.02.016.

[7] N. Degtyareva, Review of Experimental Studies of Cold-Formed Steel Channels with Slotted Webs under Bending, Procedia Engineering. 206 (2017) 875-880. doi:10.1016/j.proeng.2017.10.566.

[8] P. Keerthan, D. Hughes, M. Mahendran, Experimental studies of hollow flange channel beams subject to combined bending and shear actions, Thin-Walled Structures. 77 (2014) 129-140. doi:10.1016/j.tws.2013.12.003.

[9] P. Keerthan, M. Mahendran, D. Hughes, Numerical studies and design of hollow flange channel beams subject to combined bending and shear actions, Engineering Structures. 75 (2014) 197-212. doi:10.1016/j.engstruct.2014.05.022. 
[10] P. Keerthan, M. Mahendran, Experimental investigation and design of lipped channel beams in shear, Thin-Walled Structures. $86 \quad$ (2015) 174-184. doi:10.1016/j.tws.2014.08.024.

390

391

392

393

394

[11] C. Pham, G. Hancock, Experimental Investigation of High Strength Cold-Formed C Sections in Combined Bending and Shear, Journal Of Structural Engineering. 136 (2010) 866-878. doi:10.1061/(asce)st.1943-541x.0000172..

[12] P. Keerthan, D. Hughes, M. Mahendran, Behaviour of litesteel beams subject to combined shear and bending actions, in: 21St International Specialty Conference On Cold-Formed Steel Structures, St. Louis, Missouri, USA, 2012.

[13] P. Keerthan, H. David, M. Mahendran, Test of litesteel beams subject to combined bending and shear actions, in: Australian Structural Engineering Conference 2012 (ASEC2012), Perth, Australia, 2012.

[14] ANSYS, Commands Reference, Elements Reference, Operations Guide, Basic Analysis Guide, Theory reference for ANSYS.

[15] C. Pham, G. Hancock, Numerical simulation of high strength cold-formed purlins in combined bending and shear, Journal of Constructional Steel Research. 66 (2010) 1205-1217. doi:10.1016/j.jcsr.2010.04.014.

[16] Standards Australia/Standards New Zealand, "Australia/New Zealand Standard AS/NZS 4600 Cold-formed steel structures”, Sydney, Australia, 2005.

[17] American Iron and Steel Institute (AISI), "Specifications for the cold-formed steel structural members, cold-formed steel design manual", AISI S100, Washington DC, USA, 2012.

[18] C. Pham, Direct strength method (DSM) of design of cold-formed sections in shear, and combined bending and shear, PhD, The University of Sydney, 2010. 


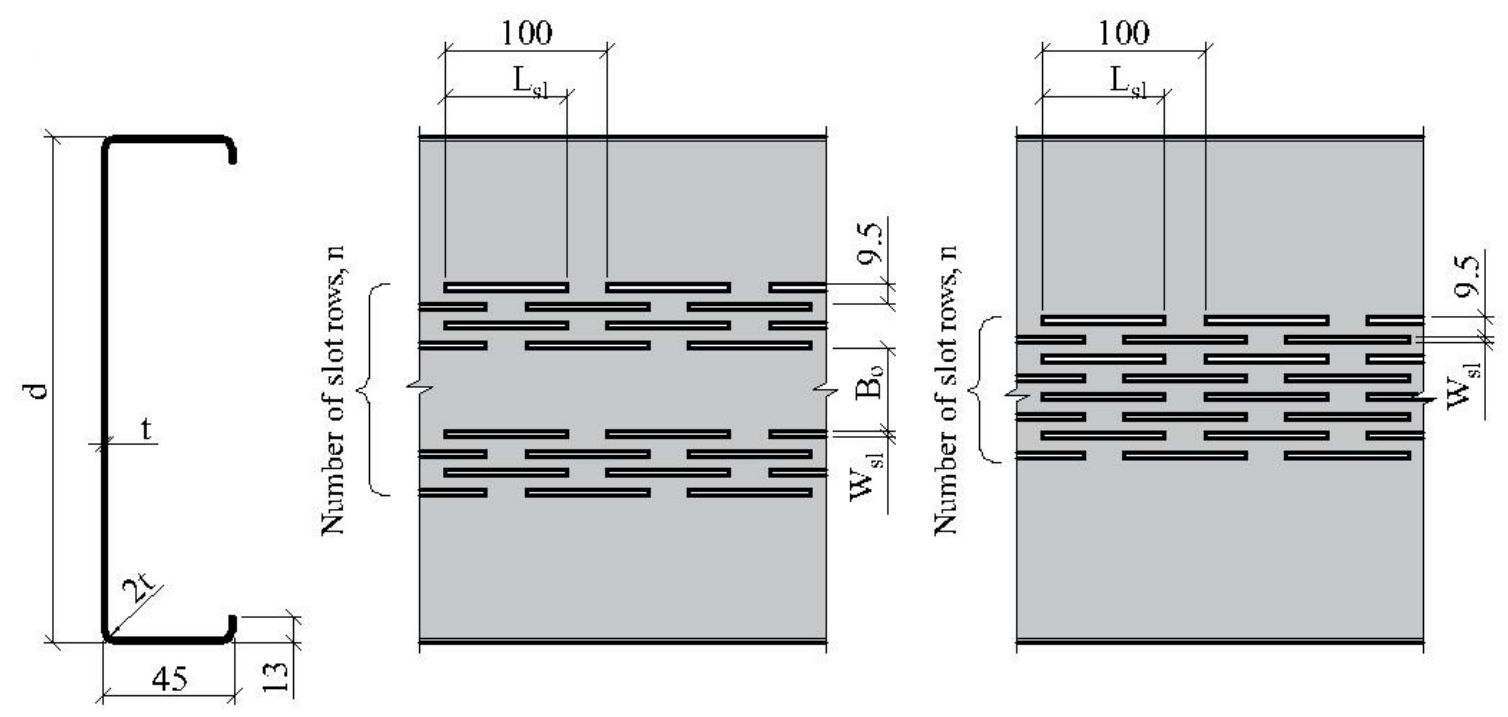

(a) Cross section

(b) Perforation pattern and sizes

Figure 1 : Cold-formed steel channels with staggered slotted perforations 

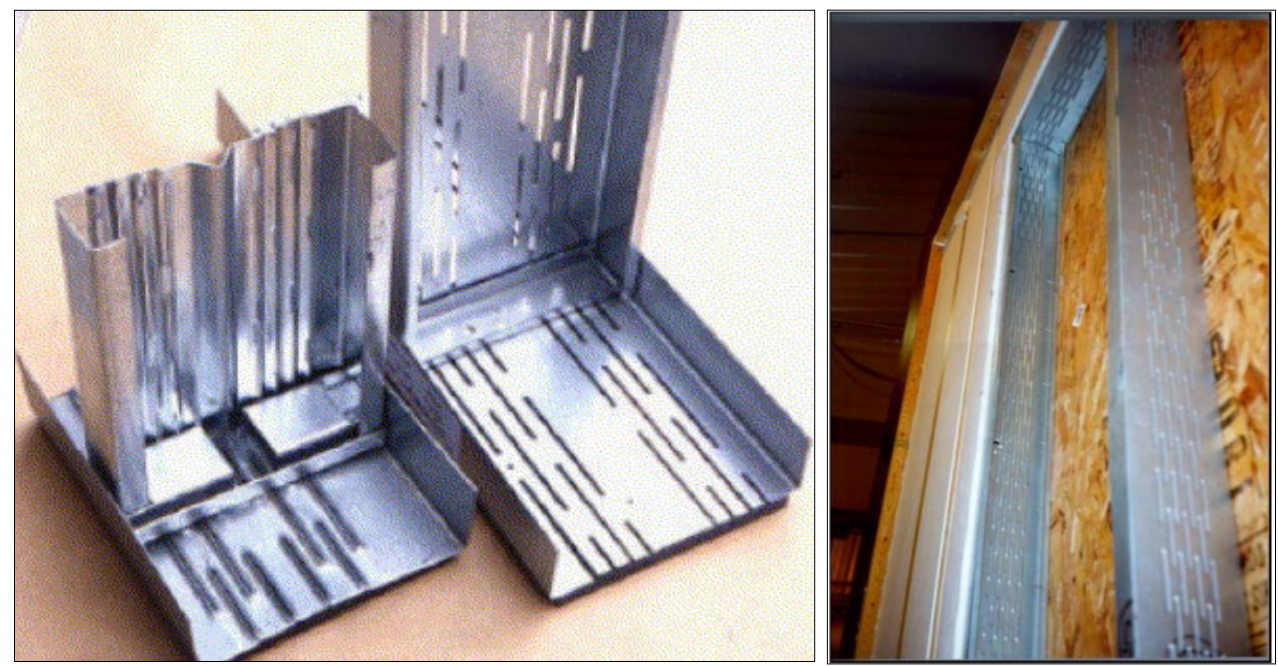

Figure 2: Application of CFS channels with slotted webs [2] 


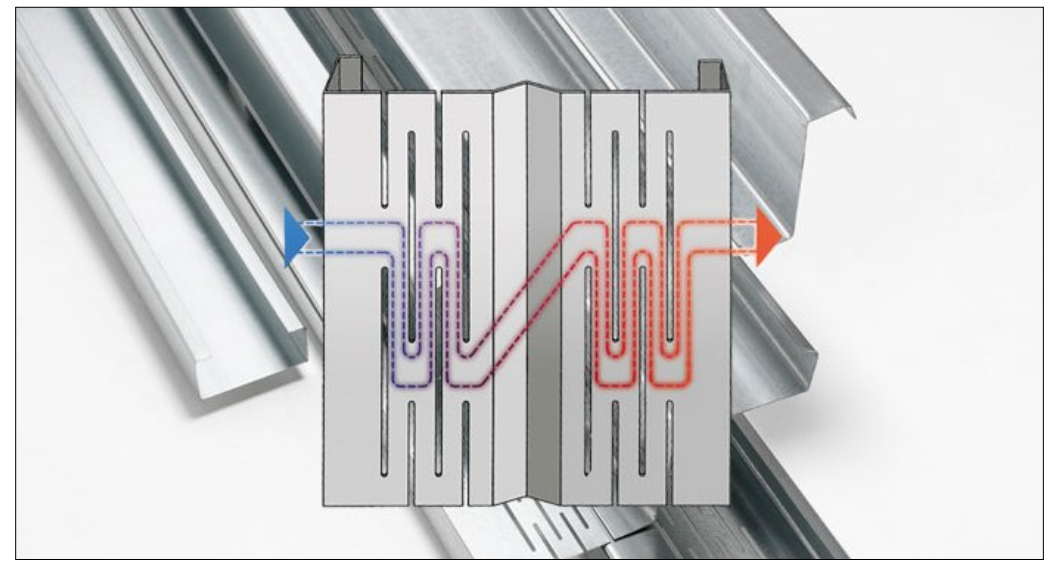

Figure 3: Thermal transmittance in wall sections with slotted steel sections 

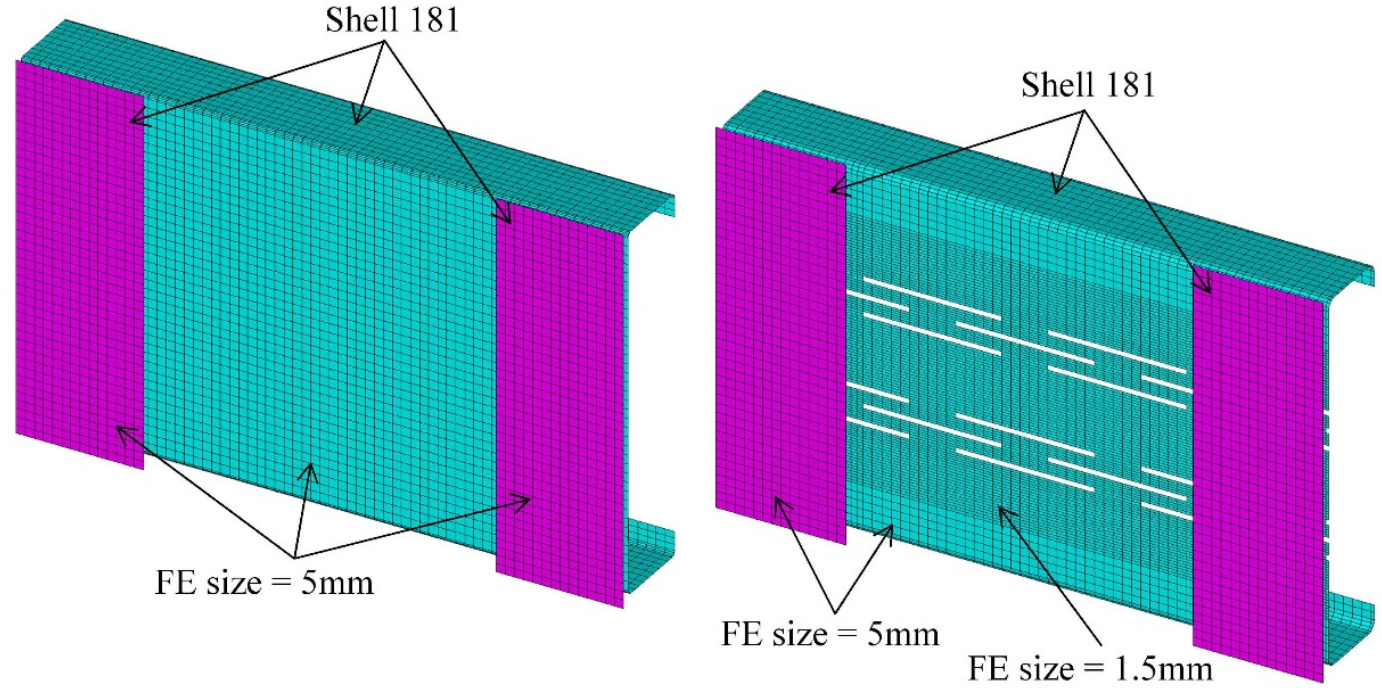

Figure 4: FE models of solid and slotted channels 


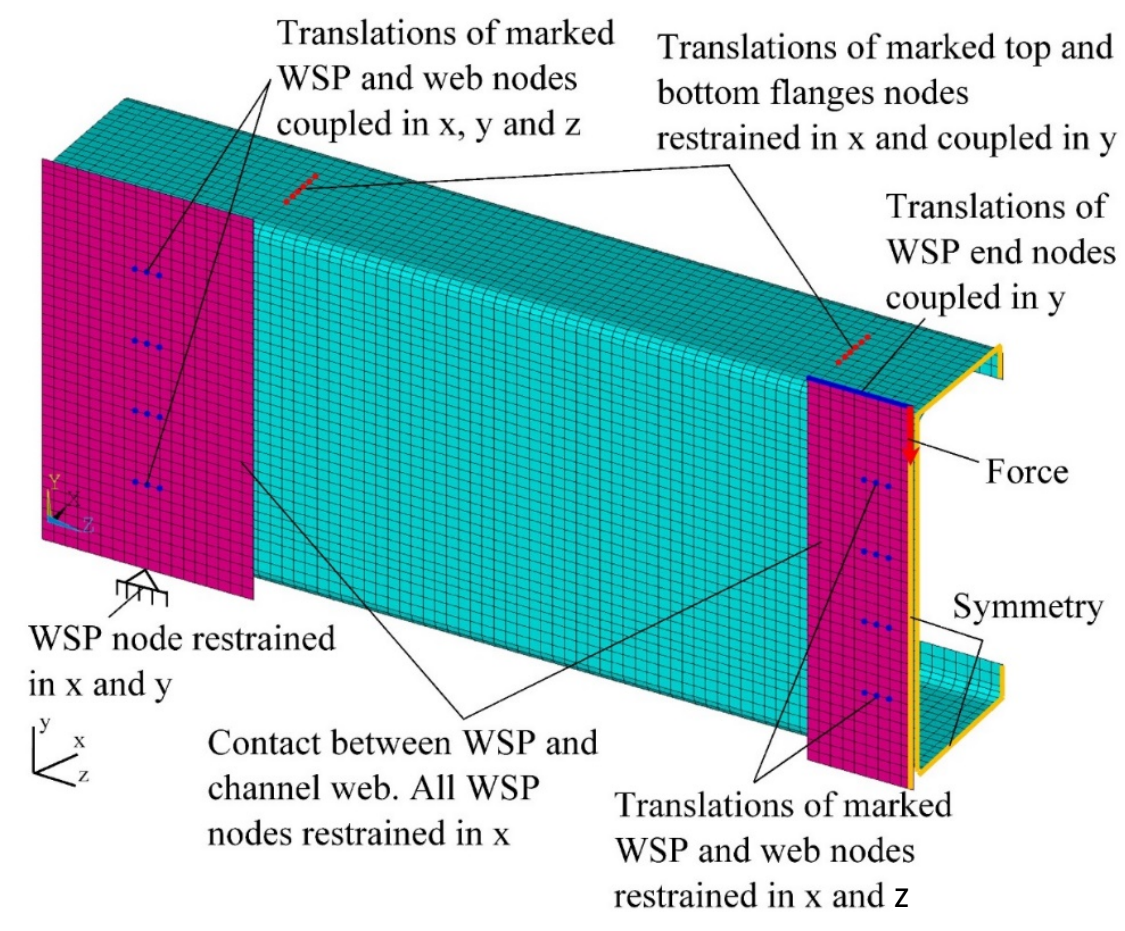

Figure 5: FE model and boundary conditions of solid channels for validation 


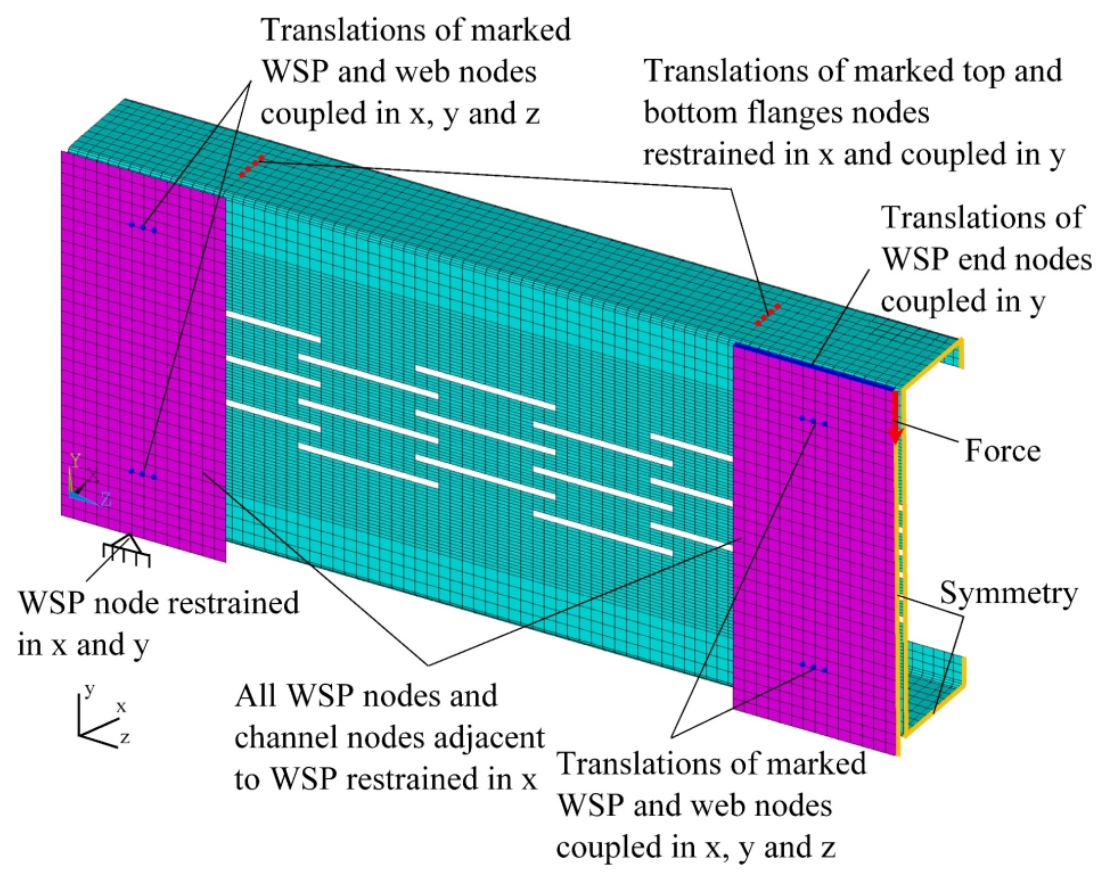

Figure 6: FE model and boundary conditions of channels with the slotted web for parametric study 


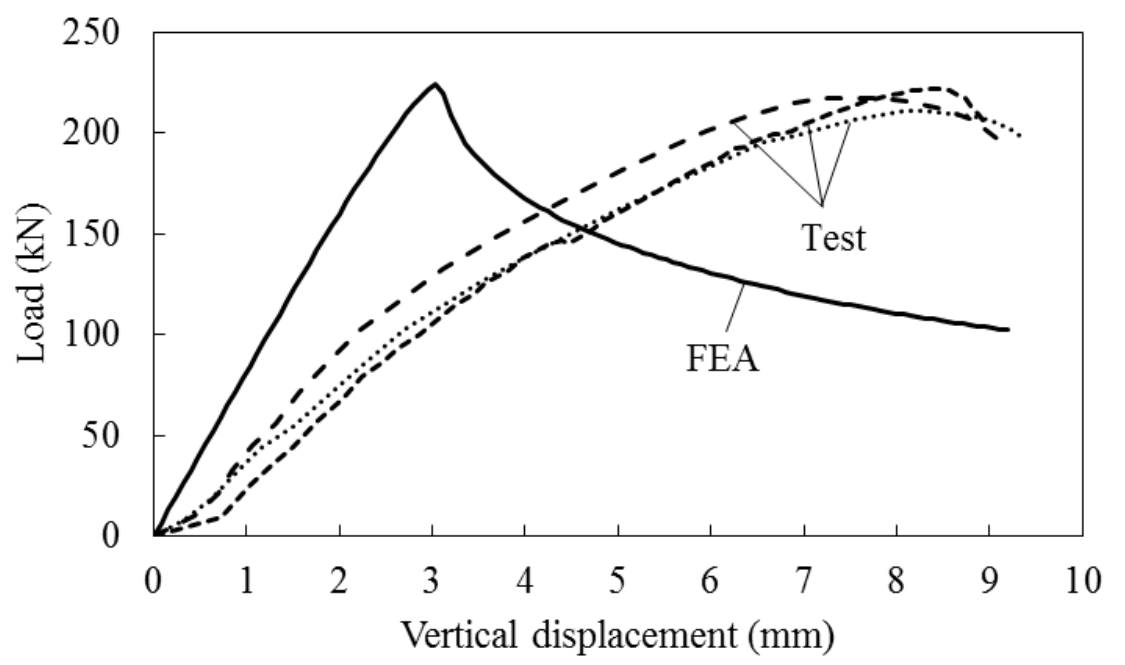

Figure 7: Load-vertical displacement behaviours for $200 \mathrm{~mm}$ deep channel with a thickness of $1.9 \mathrm{~mm}$ with straps (C20019-MVl). 


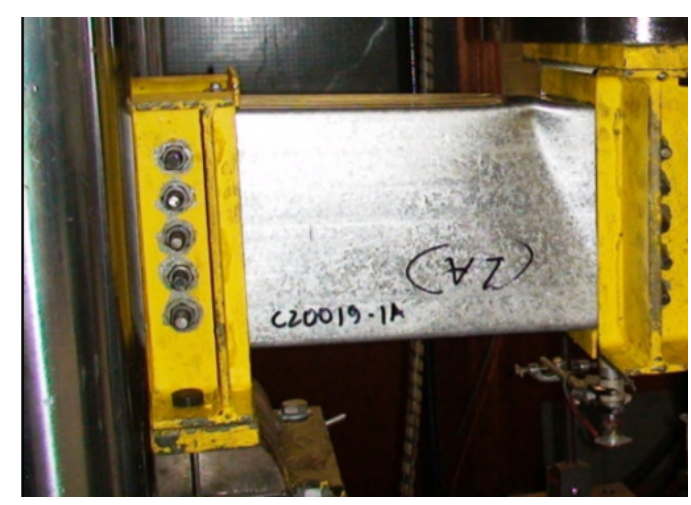

(a) Test

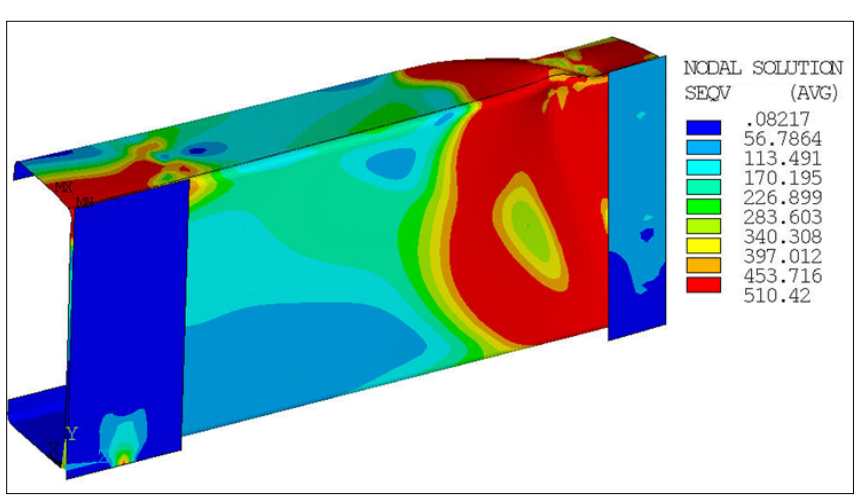

(b) FE analysis

Figure 8: Failure modes shapes of the tested specimen [15] and FE model of $200 \mathrm{~mm}$ deep channel with a thickness of $1.9 \mathrm{~mm}$ and with straps (C20019-MVl) 


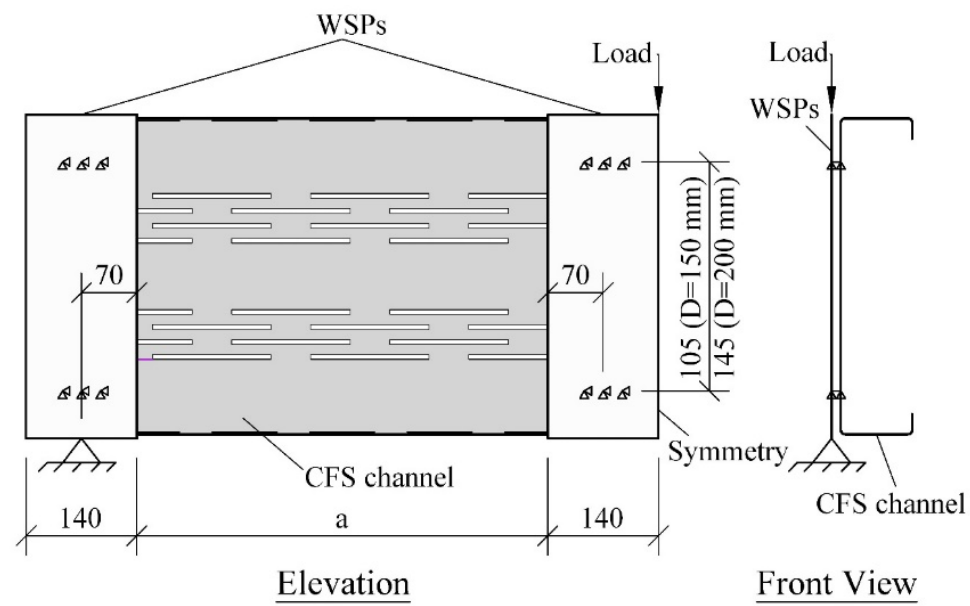

Figure 9: Dimensions of FE model of channels for parametric study 


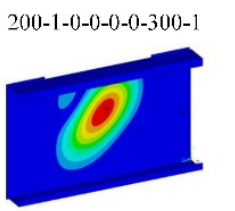

$200-1-75-3-1-8-300-1 \quad 200-1-75-3-1-8-300-1.5$

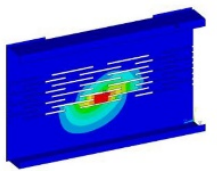

$200-1-75-3-2-8-300-$
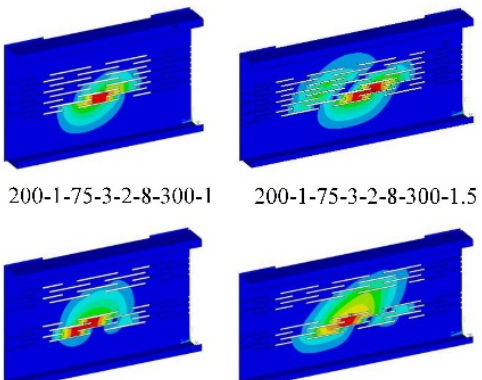

$200-1-75-3-2-8-300-1.5$

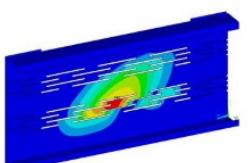

$200-1-0-0-0-0-300-2$

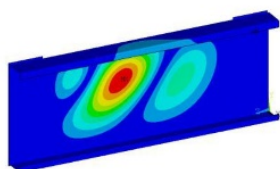

$200-1-75-3-1-8-300-2$

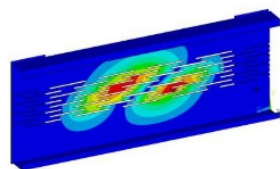

$200-1-75-3-2-8-300-2$

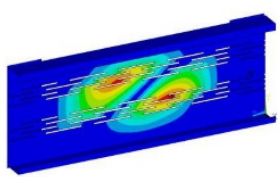

$200-1-0-0-0-0-300-3$

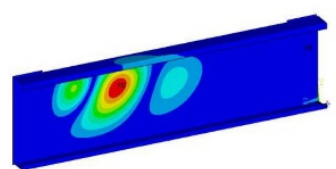

200-1-75-3-1-8-300-3

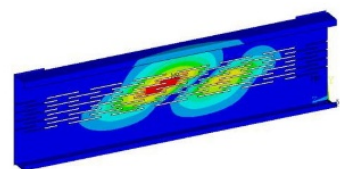

$200-1-75-3-2-8-300-3$

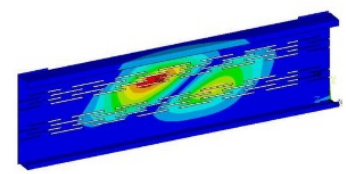

$200-1-0-0-0-0-300-4$

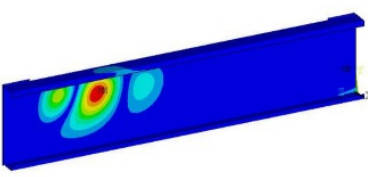

$200-1-75-3-1-8-300-4$

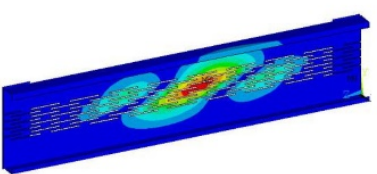

$200-1-75-3-2-8-300-4$

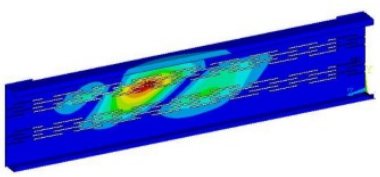

Figure 10: Critical buckling mode of solid and slotted channels subjected to combined bending and shear 

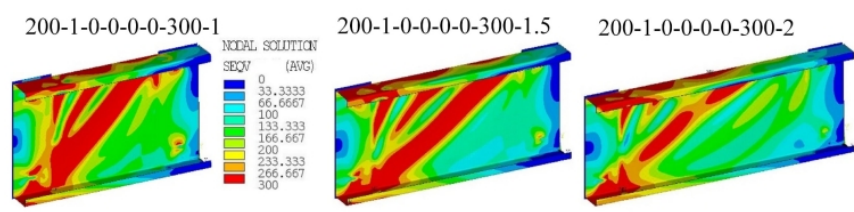

$200-1-0-0-0-0-300-3$

$200-1-0-0-0-0-300-4$
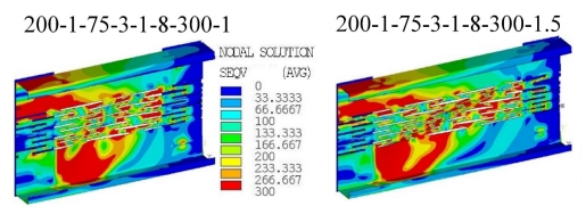

$200-1-75-3-1-8-300-2$

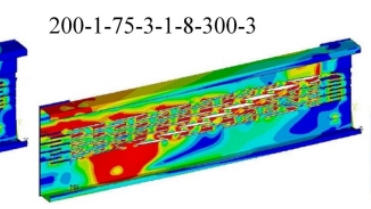

$200-1-75-3-1-8-300-4$
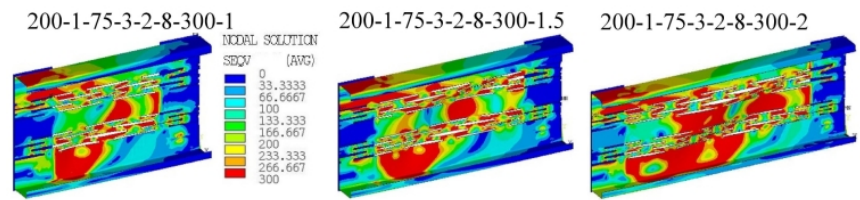

$200-1-75-3-2-8-300-3$

Figure 11: Von misses stresses in solid and slotted channels at maximum applied load 


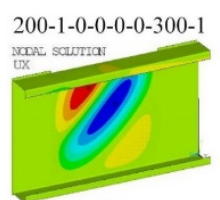

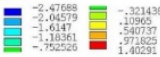

200-1-75-3-1-8-300-

INOAL, soumtar

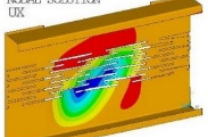

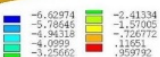

$200-1-75-3-2-8-300-1$ mont sowntai

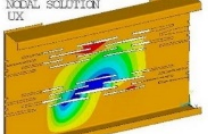

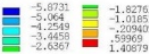

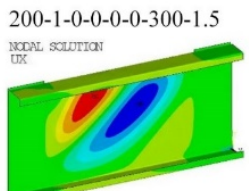

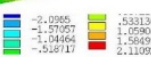

200-1-75-3-1-8-300-1.5
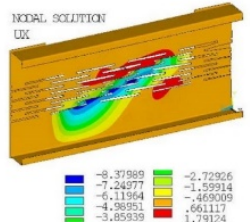

200-1-75-3-2-8-300-1.5

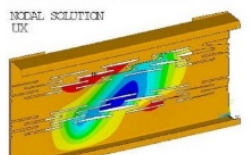

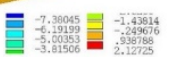

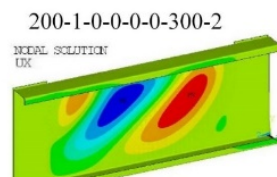

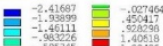

200-1-75-3-1-8-300-2

NMOAL scaurtias

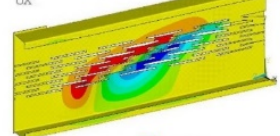

-1)

200-1-75-3-2-8-300-2

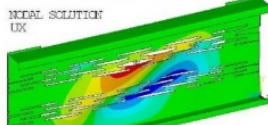

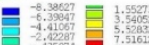

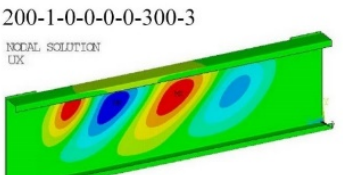

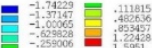

$200-1-75-3-1-8-300-3$

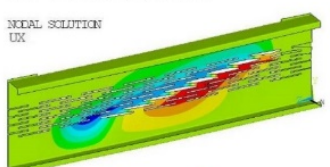

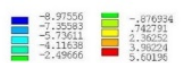

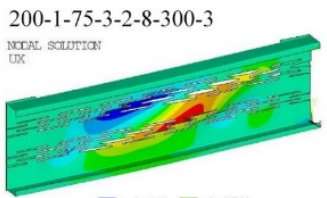

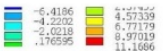

200-1-0-0-0-0-300-4

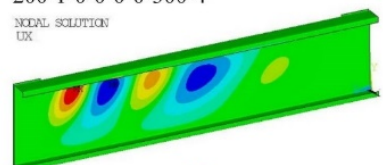

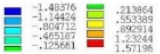

200-1-75-3-1-8-300-4

MDOA saurra:

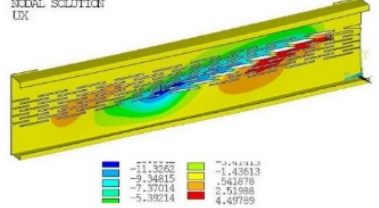

200-1-75-3-2-8-300-4

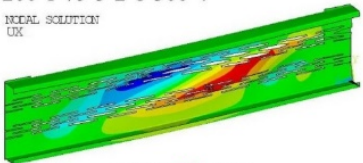

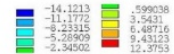

Figure 12: Failure modes in solid and slotted channels at maximum applied load 

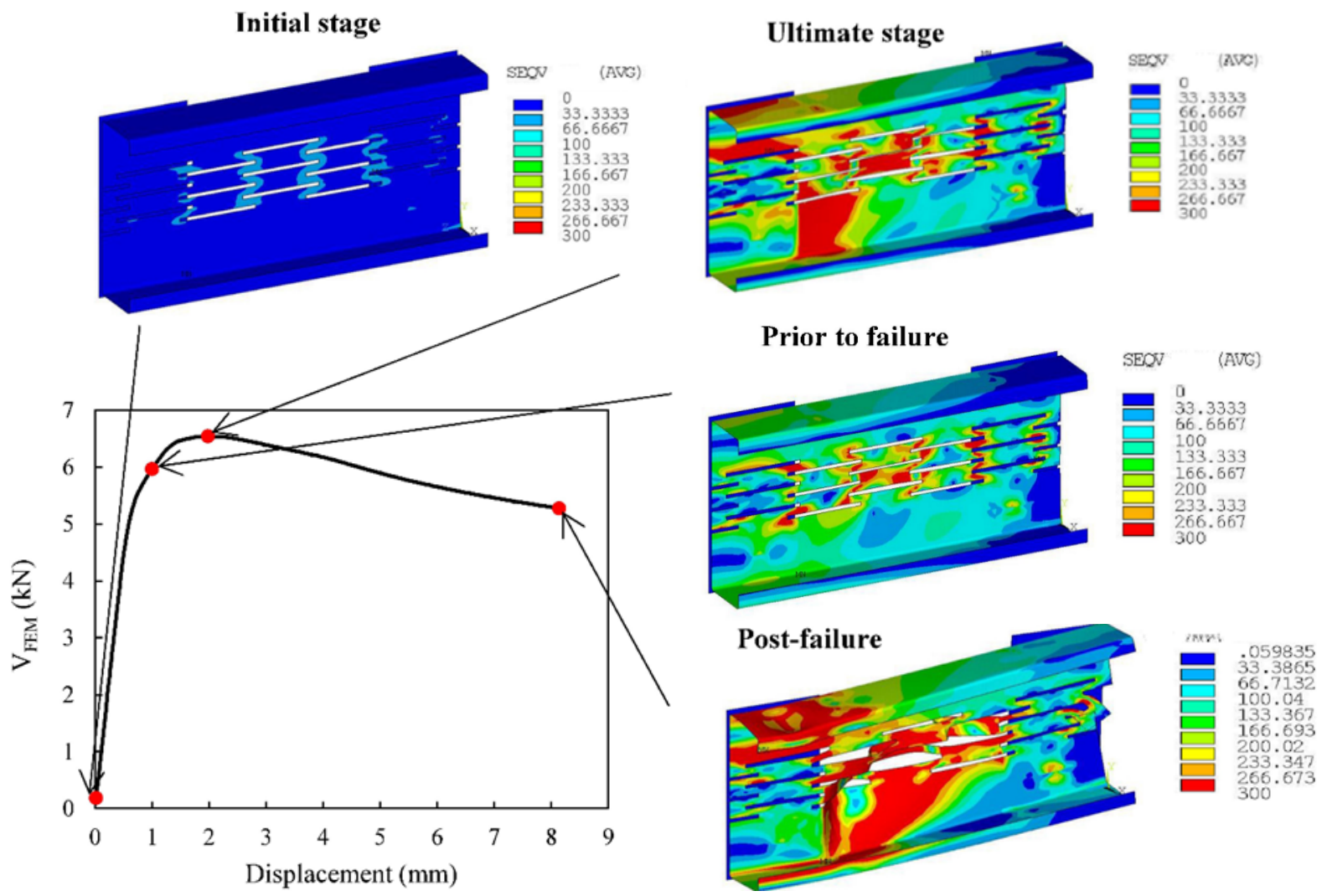

Figure 13: Load-deflection plot of slotted channel 150-1-60-3-1-6-300-1 and its behavior at different stages 


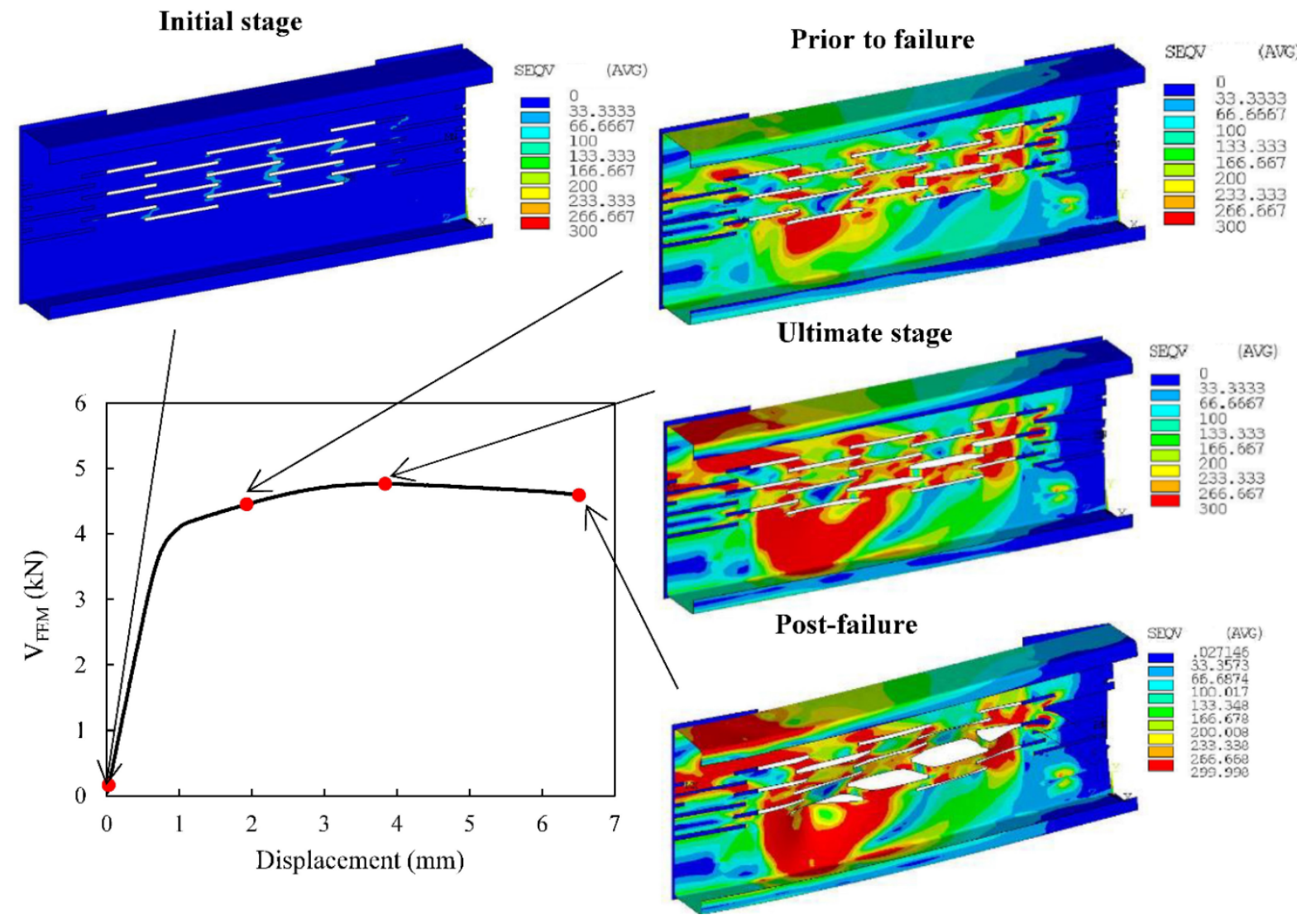

Figure 14: Load-deflection plot of slotted channel 150-1-60-3-1-6-300-1.5 and its behavior at different stages 


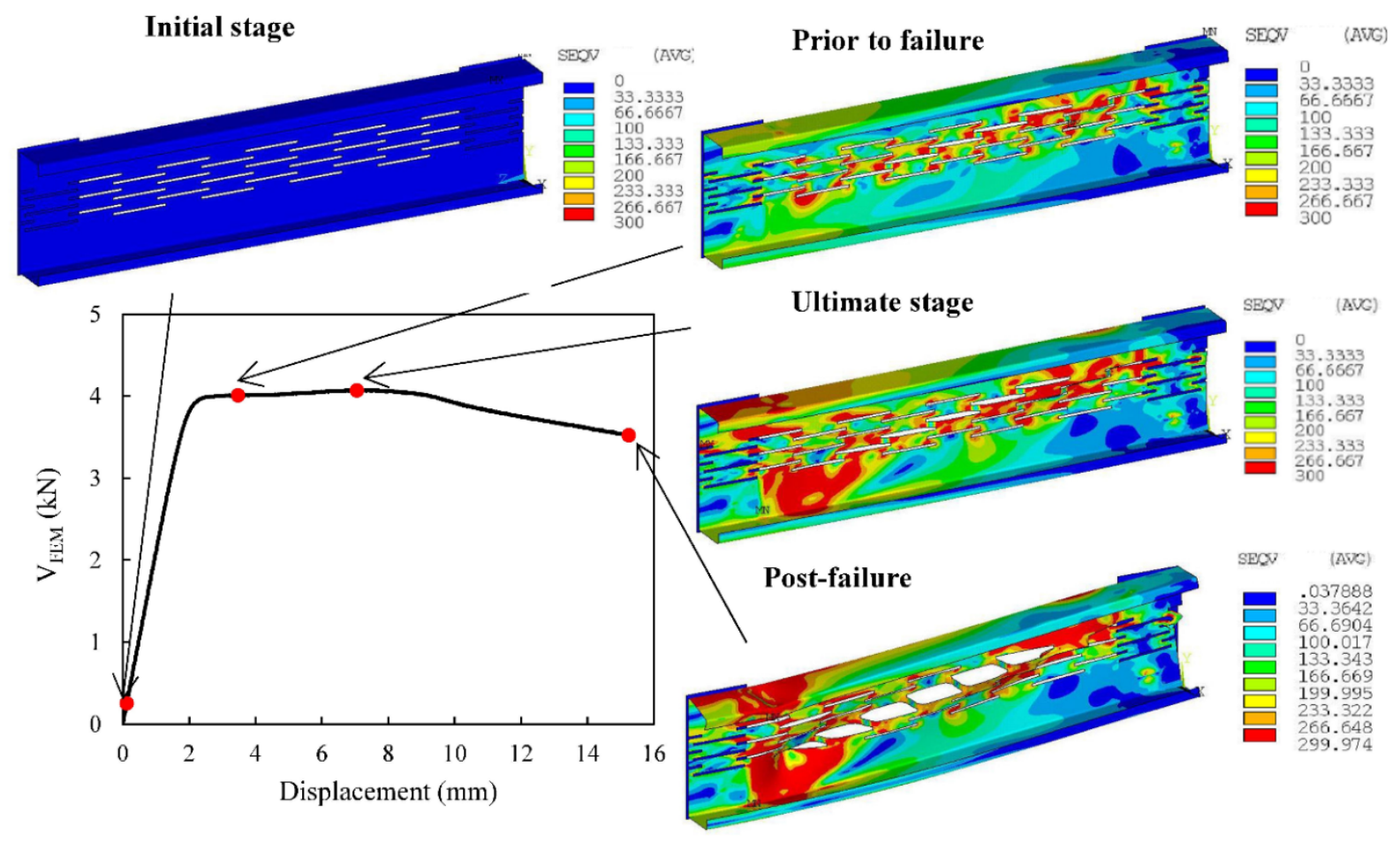

Figure 15: Load-deflection plot of slotted channel 150-1-60-3-1-6-300-3 and its behavior at different stages 


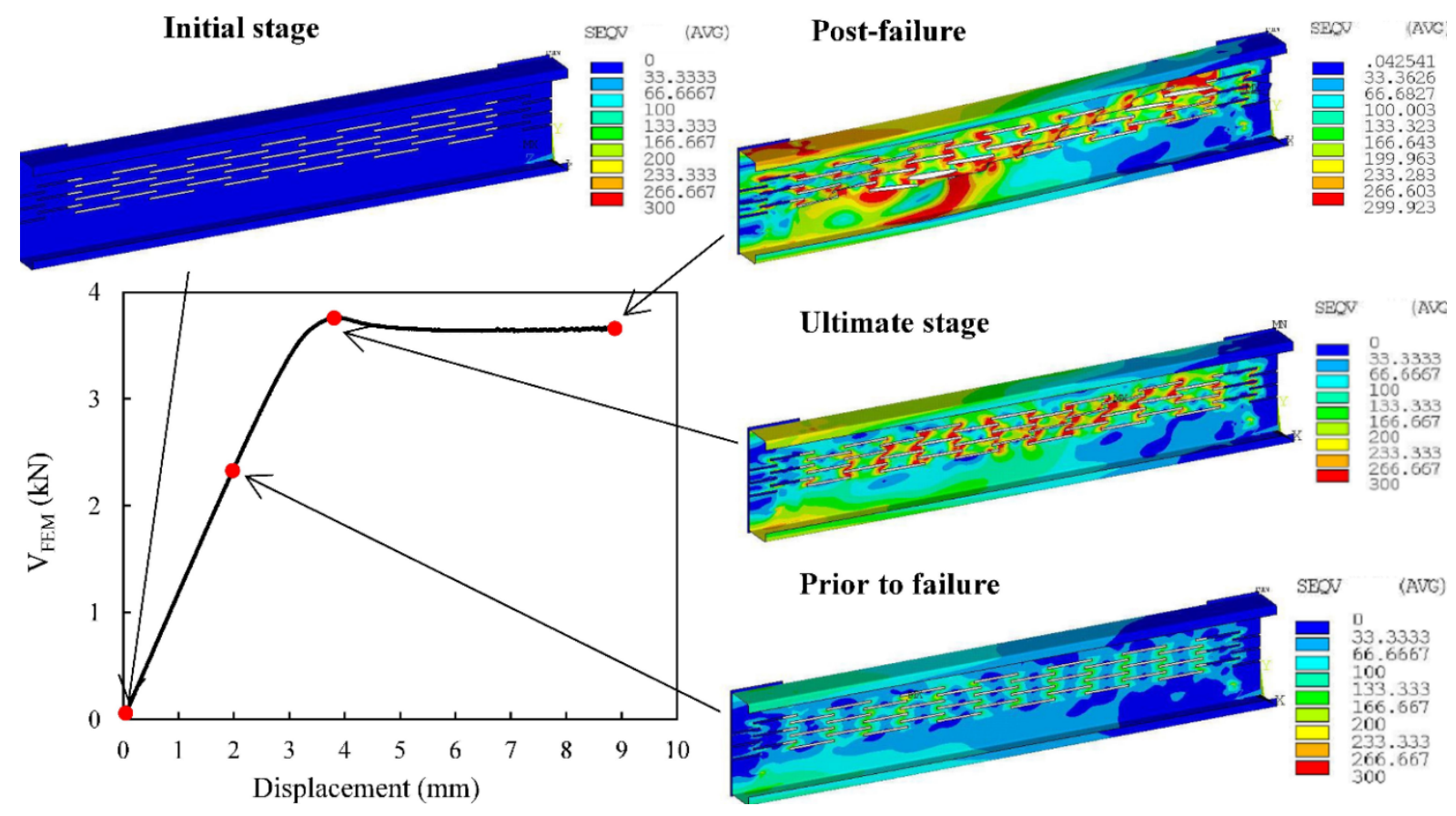

Figure 16: Load-deflection plot of slotted channel 150-1-60-3-1-6-300-4 and its behavior at different stages 


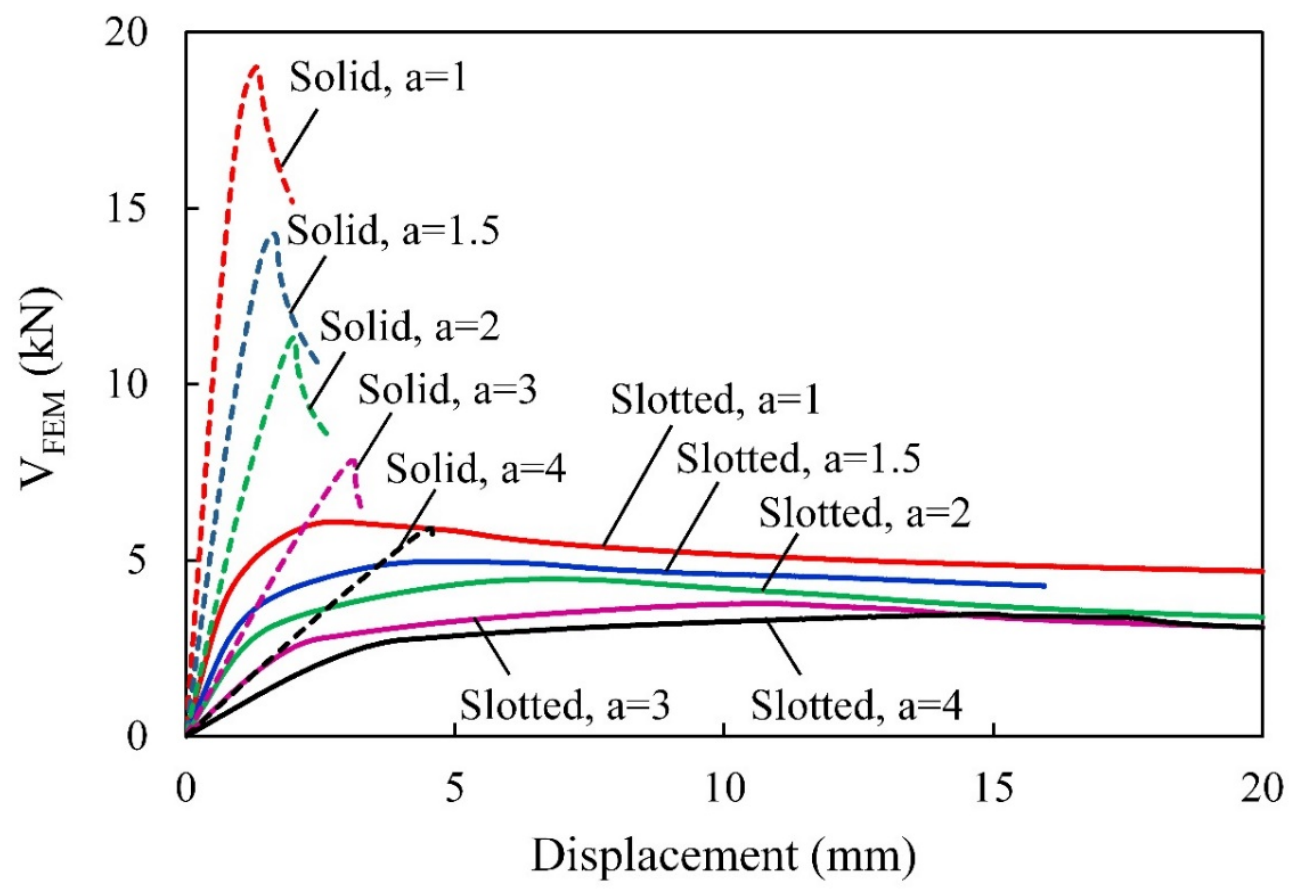

Figure 17: Load-deflection plots of solid (200-1-0-0-0-0-300) and slotted (200-1-75-3-1-8-300) channels with different aspect ratios 


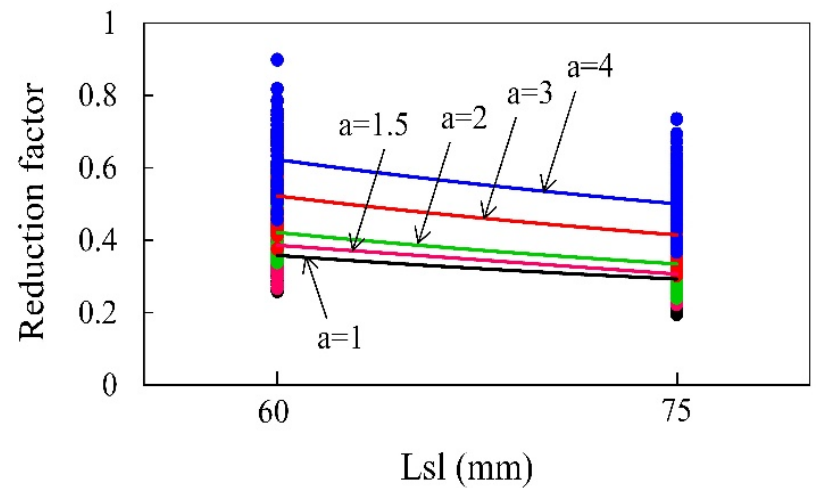

(a) Slot length $\left(\mathrm{L}_{\mathrm{sl}}\right)$

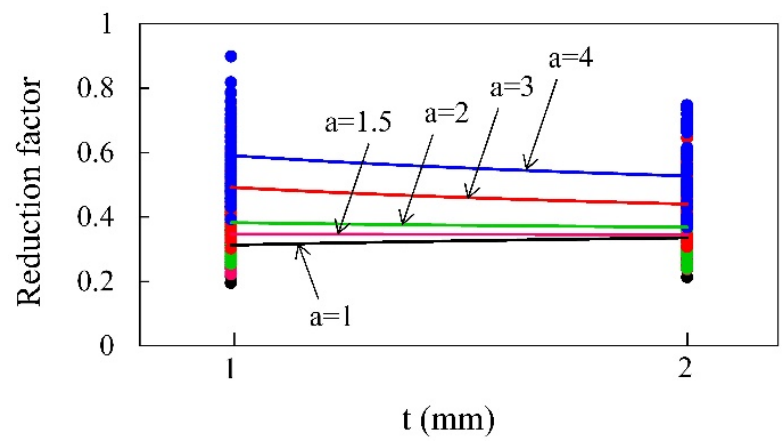

(c) Thickness (t)

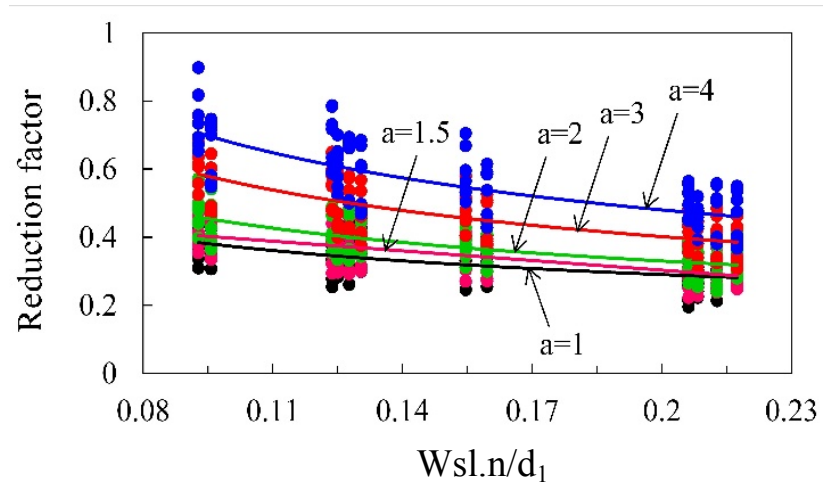

(b) Slot width $\left(\mathrm{W}_{\mathrm{sl}}\right)$

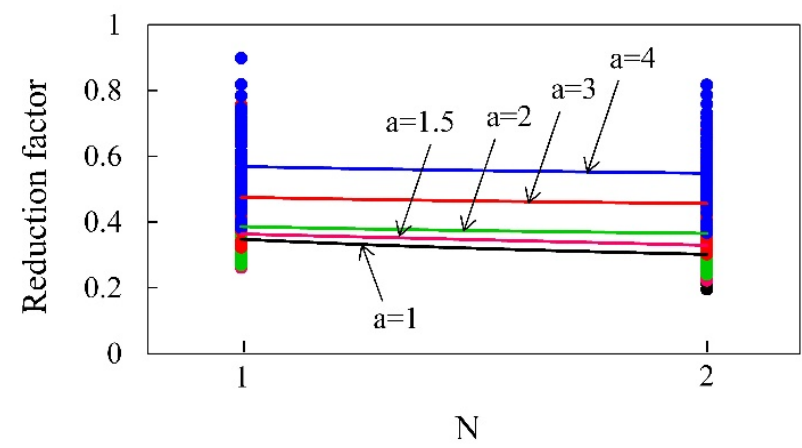

(d) Number of slot row groups (N)

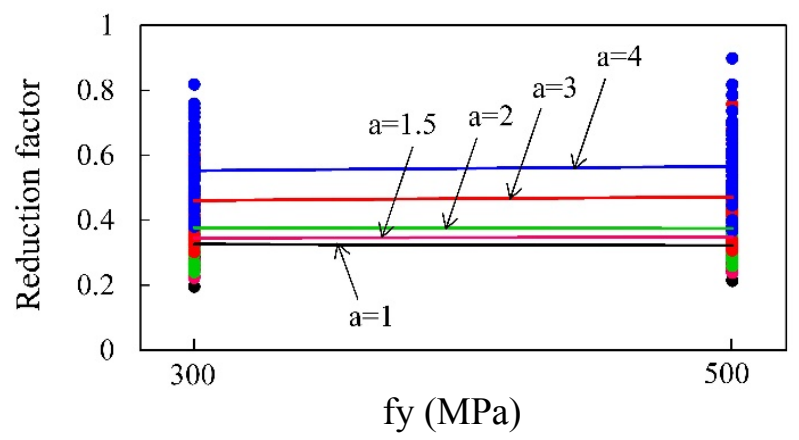

(e) Yield strength (fy)

Figure 18: Variation of reduction factor with influencing parameters. 


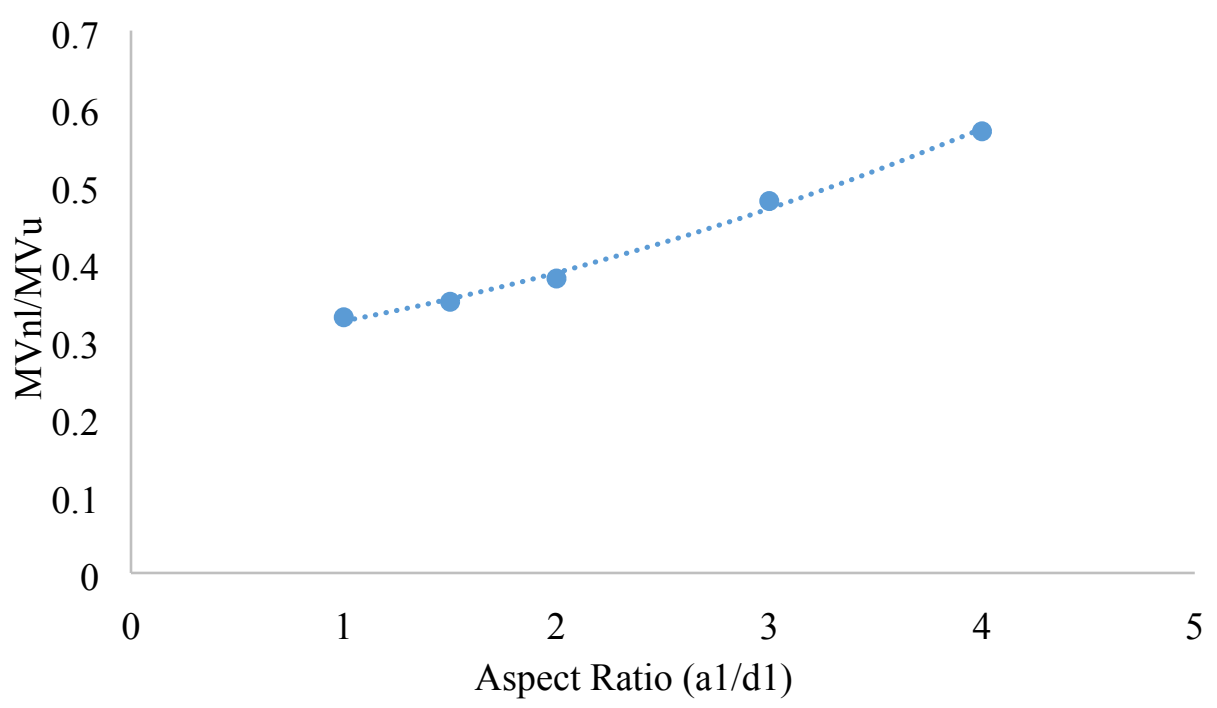

Figure 19: Plot of reduction factor $\left(M V_{n} / M V_{u}\right)$ when aspect ratio $\left(a_{1} / d_{1}\right)$ is only considered. 


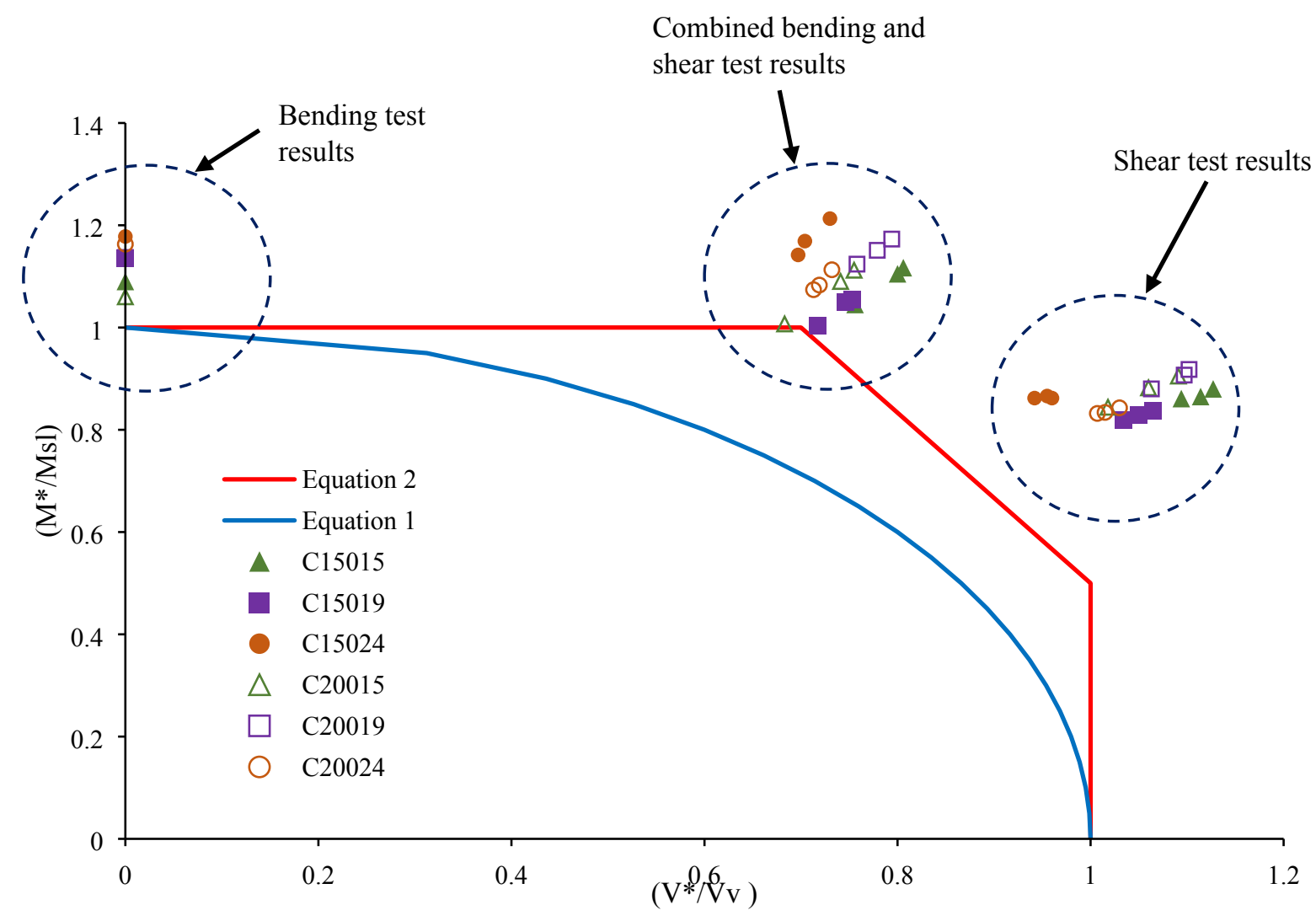

Figures 20: Interaction between $\left(\mathrm{M}^{*} / \mathrm{M}_{\mathrm{sl}}\right)$ and $\left(\mathrm{V}^{*} / \mathrm{V}_{\mathrm{v}}\right)$ (both $\mathrm{Msl}$ and $\mathrm{Vv}$ are based on direct strength method)[18] 


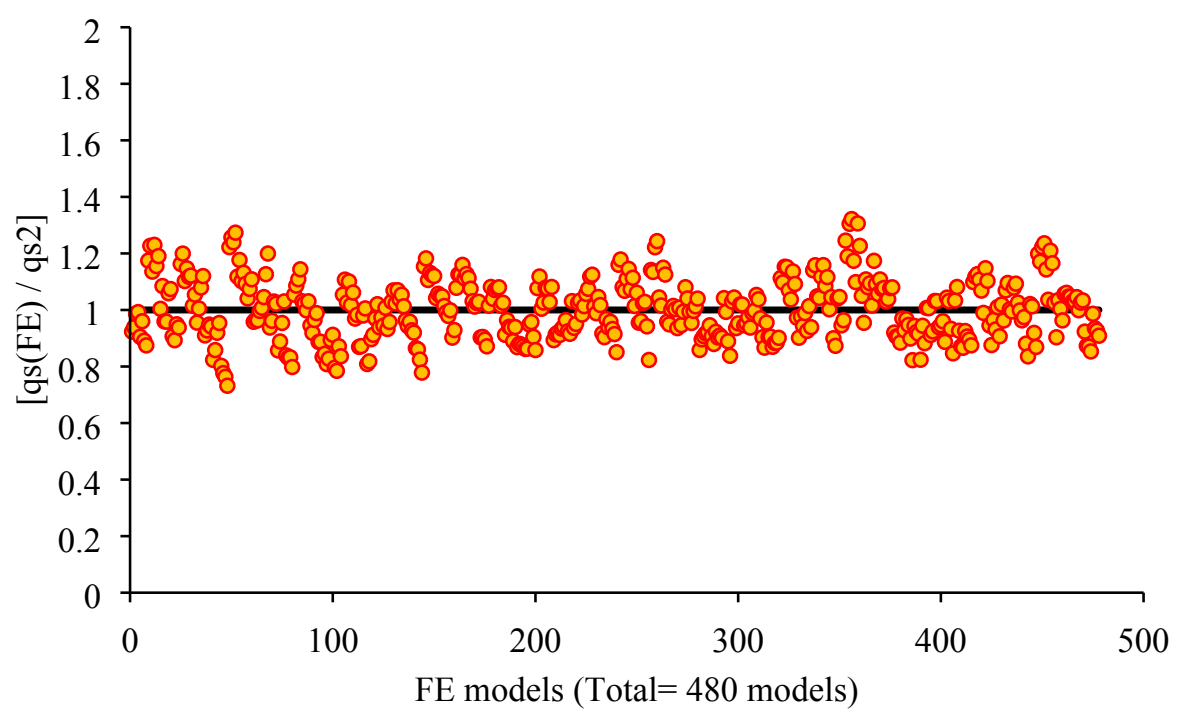

Figure 21: Ratio between reduction factors obtained from FE analysis and proposed equation 


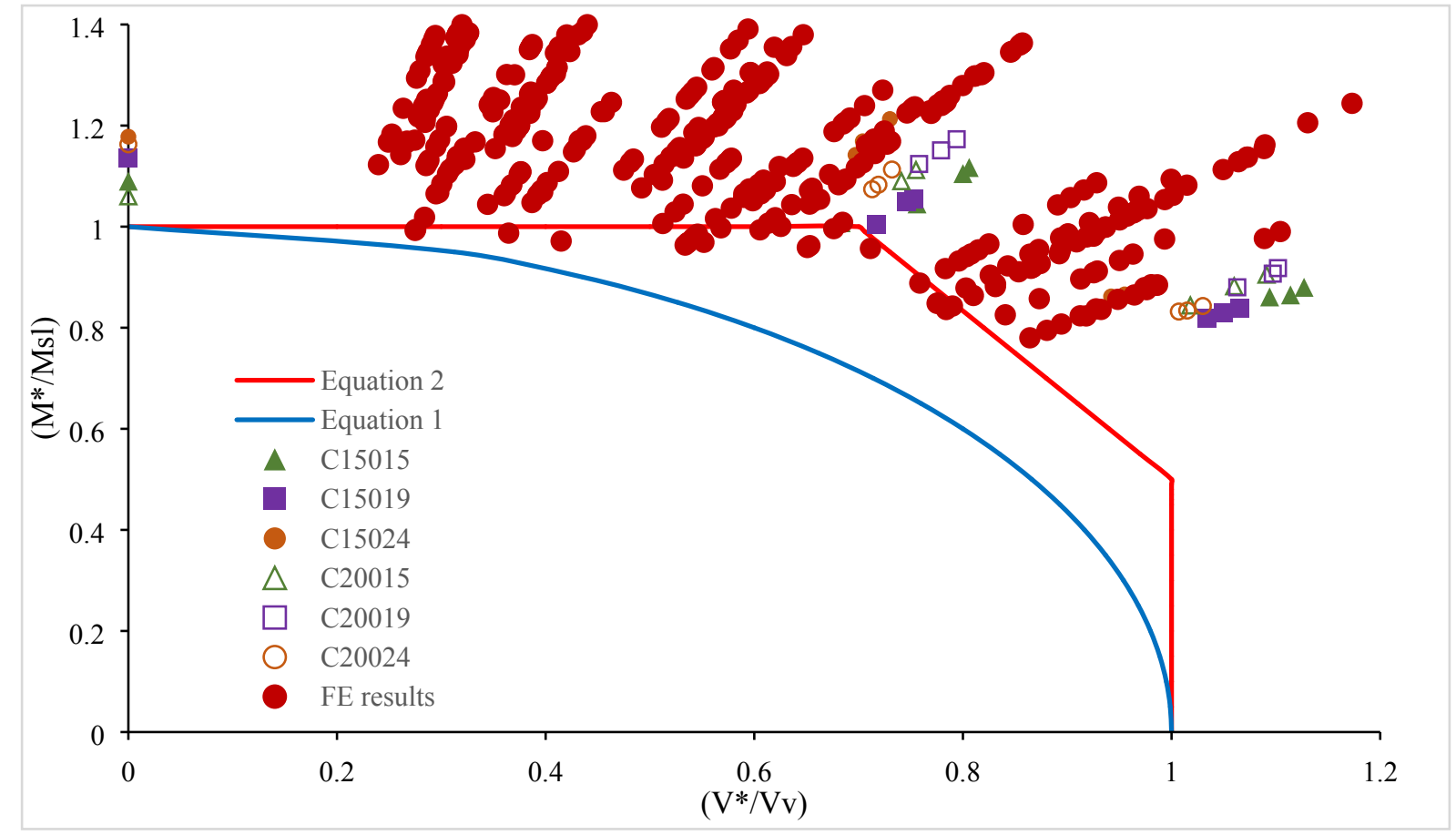

Figures 22: FE results of the 480 models along with test [18] results 